\title{
AKARI All Sky Survey: contribution from AGB stars to the far infrared flux from the Milky Way related to point sources outside the Galactic plane
}

\author{
Agnieszka Pollo ${ }^{1,2,3}$, Tsutomu T. Takeuchi ${ }^{4,5}$, and Piotr Rybka ${ }^{2,5}$ \\ ${ }^{1}$ The Andrzej Sottan Institute for Nuclear Studies, ul. Hoża 69, 00-681 Warszawa, Poland \\ ${ }^{2}$ Astronomical Observatory of the Jagiellonian University, ul. Orla 171, 30-244 Kraków, Poland \\ ${ }^{3}$ Center for Theoretical Physics PAS, Al. Lotników 32/46, 02-668 Warszawa, Poland \\ ${ }^{4}$ Institute for Advanced Research, Nagoya University, Furo-cho, Chikusa-ku, Nagoya 464-8601, Japan \\ ${ }^{5}$ Division of Particle and Astrophysical Science, Nagoya University, Furo-cho, Chikusa-ku, Nagoya 464-8602, Japan
}

(Received September 14, 2010; Revised April 13, 2011; Accepted April 15, 2011; Online published February 2, 2012)

\begin{abstract}
Using data from the FIS AKARI All-Sky Survey, we make a first step towards the estimation of the contribution from Asymptotic Giant Branch (AGB) stars to the far-infrared (FIR) flux from the Milky Way. We estimate the contribution from the AGB, and post-AGB, stars to the total flux generated by point sources outside the Galactic plane. Additionally, we present the positions of different types of AGB, and post-AGB, stars in the FIR colorcolor diagrams. Our main conclusion is that there is a high contribution from AGB stars, and particularly postAGB stars, to the FIR flux coming from point sources in the outer parts of the Milky Way and possibly other Milky Way-type galaxies. FIR colors of different types of AGB stars remain similar but post-AGB stars are redder in the FIR and, as a result, contribute more to the total Galaxy flux density at longer FIR wavelengths.
\end{abstract}

Key words: Dust, AGB stars, post-AGB stars, far-infrared, all-sky surveys.

\section{Introduction}

Emission from galaxies at wavelengths longer than a few micrometers is produced mainly by dust. It is often assumed that dust emission is associated predominantly with star formation (e.g., Dwek, 1998; Pipino et al., 2011; Asano et al., 2012). Indeed, as is widely known, star-formation activity is always accompanied by dust production on a timescale of $10^{6} \mathrm{yr}$, very probably through dust production in supernova explosion (e.g., Kozasa and Hasegawa, 1987; Kozasa et al., 1991; Nozawa et al., 2003, 2007, 2008, 2010), though the actual mechanism of dust supply has not been fully understood yet. In such a case, the energy emitted by massive stars in the ultraviolet (UV) is scattered efficiently and finally absorbed by dust grains. The heated grains re-emit the energy in the far-infrared (FIR). Consequently, radiative processes in FIR can be related both to the composition and the amount of dust in galaxies and to the properties of their stellar population, especially the fraction of newly-formed massive stars.

Many studies have shown that a significant amount of star formation in galaxies is obscured by dust (Le Floc'h et al., 2005; Caputi et al., 2007; Buat et al., 2007a, b, 2008; Reddy et al., 2008). It has also been found that such "hidden" star formation becomes more and more important with increasing redshift from $z=0$ to 1 (Takeuchi et al., 2005). Therefore, FIR observations are of crucial importance to understand the true star-formation activity and history in the Uni-

Copyright (c) The Society of Geomagnetism and Earth, Planetary and Space Sciences (SGEPSS); The Seismological Society of Japan; The Volcanological Society of Japan; The Geodetic Society of Japan; The Japanese Society for Planetary Sciences; TERRAPUB.

doi:10.5047/eps.2011.04.005 verse.

In the context of star-formation activity, supernovae ( $\mathrm{SNe}$ ) are often discussed as a source of dust supply, because $\mathrm{SNe}$ are the final stage of the evolution of massive $\left(M>8 M_{\odot}\right)$ stars that are the tracers of star-formation history, since their lifetime is very short (a few Myr). Up to now, the quantity of dust produced by a supernova is a matter of debate, because theories tend to predict a high efficiency of SN dust production $\left(0.1-1 M_{\odot}\right.$ per core-collapse SN: e.g., Kozasa et al., 1991; Clayton et al., 2001; Nozawa et al., 2003) while a smaller amount is inferred from observations (e.g., Rho et al., 2008, 2009), but the discrepancy between theory and observation is now diminishing.

By contrast, in present-day evolved galaxies, the major sources of dust are stars in the asymptotic giant branch (AGB), and post-AGB, phases. The AGB is the final phase of evolved intermediate-mass $\left(0.8 M_{\odot}<M<8 M_{\odot}\right)$ stars, expelling dust into the interstellar medium (ISM) via planetary nebulae. In the context of this work, since both AGB, and post-AGB, stars expel gas and associated dust by superwind phenomena, we say they are a source of dust, though a detailed mechanism is still not perfectly clarified. Further, the mass contribution of dust from AGB-related phenomena has been proved to be significant even $~ 500 \mathrm{Myr}$ after the birth of a galaxy (Valiante et al., 2009; Asano et al., 2010). Some recent studies claim that the contribution to the mid-far-IR luminosity is also very important (Kelson and Holden, 2010; Takeuchi et al., 2010). Thus, a proper understanding of dust produced by AGB stars, as well as $\mathrm{SN}$-related dust, is crucial for interpreting dust emission at any age of the Universe.

In order to estimate the contribution from each com- 
ponent of dust emission, all-sky far-IR survey data are desirable. The Infrared Astronomical Satellite (IRAS; Neugebauer et al., 1984) has brought a vast amount of statistics and very efficient methods of analysis have been devised from the IRAS Point Source Catalog (PSC). The four bands of IRAS have enabled us even to perform a very detailed classification of extragalactic and various galactic objects, like blue and red galaxies, Seyferts and QSOs, carbon stars, HII regions, reflection nebulae, planetary nebulae, T Tauri stars, etc. Hacking et al. (1985) classified the stars on the IRAS color-color plane, and found that C- and Orich stars occupy a separated region different from that for normal stars. Thronson et al. (1987) showed a distinction between C- and O-rich stars based on IRAS colors. Van der Veen and Habing (1988) introduced ten zones for the detailed classification of IRAS-detected stars, which was later extensively used by Busso et al. (1996). Walker and Cohen (1988) also defined occupation zones for stars. A thorough description of the IRAS color-color classification method including extragalactic objects and star-forming regions can be found in, e.g., Walker et al. (1989).

After many years since IRAS, the advent of AKARI (ASTRO-F) opened a new window to explore the Universe, as a survey-oriented space telescope at MIR and FIR (Murakami et al., 2007). The primary purpose of the AKARI mission is to provide second-generation infrared (IR) catalogs to obtain a better spatial resolution and a wider spectral coverage than the IRAS catalog. Using the AKARI FIS All-Sky Survey Bright Source Catalog and existing databases (NED and SIMBAD), we have constructed a method to classify the sources on the color-color diagram only from FIS bands (Pollo et al., 2010a). In Pollo et al. (2010a), we found that it is quite plausible to select AGB stars from the FIS catalog exclusively. The method itself remains to be improved by a more sophisticated examination, but this is to be done in our future work (Rybka et al., 2011 , in preparation).

As an interesting comparison, we note that Ita et al. (2010) showed color-color diagrams of nearby stars from the AKARI IRC All-Sky Survey. Also, recently, Phillips and Marquez-Lugo (2011) presented IRC-FIS-based colorcolor diagrams of planetary nebulae, and Cox et al. (2011) have shown AKARI images of 13 post-AGBs and (proto)planetary nebulae.

In this work, we make an attempt to quantify the contribution from AGB, and post-AGB, stars to the FIR flux related to point sources from the outer regions of the Milky Way, using data gathered by the satellite AKARI in the FIS All Sky Survey. We expect that our conclusions on the Milky Way can be also applied-with due attentionto other similar galaxies.

The article is organized as follows: in Section 2, we present the data used for this analysis and the statistics of AGB stars found in them. In Section 3, we present and discuss the location of different types of AGB stars in the FIR color-color plots. In Section 4, we discuss the contribution from AGB, and post-AGB, stars to the FIR flux of the Milky Way. Section 5 presents a summary.

\section{The Data \\ 2.1 AKARI}

AKARI is a Japanese astronomical satellite whose purpose is to perform various large-area surveys at the IR from NIR to FIR with a wavelength coverage of 2-160 $\mu \mathrm{m}$, as well as pointed observations. ${ }^{1}$ AKARI is equipped with a cryogenically-cooled telescope of $68.5 \mathrm{~cm}$ aperture diameter and two scientific instruments, the Far-Infrared Surveyor (FIS; Kawada et al., 2007) and the Infrared Camera (IRC; Onaka et al., 2007). Among various astronomical observations performed by AKARI, an all sky survey with FIS and IRC has been carried out; it is referred to as the AKARI All-Sky Survey.

Since FIS is an instrument dedicated to FIR, $\lambda=$ 50-180 $\mu \mathrm{m}$, all the AKARI FIS bands are in the FIR wavelengths: $N 60(65 \mu \mathrm{m})$, WIDE-S $(90 \mu \mathrm{m})$, WIDE-L $(140 \mu \mathrm{m})$, and N160 $(160 \mu \mathrm{m})$ (Kawada et al., 2007). Hereafter, we use a notation $S_{65}, S_{90}, S_{140}$ and $S_{160}$ for flux densities in these bands.

Especially, since FIS has a better sensitivity and reaches longer wavelengths than IRAS, we can expect a different composition of sources: we should see objects with cool dust which were difficult to detect by IRAS bands.

\subsection{Catalogs}

As mentioned above, one of the main goals of AKARI was to carry out the All-Sky Survey in the four photometric bands in the far-infrared wavelength range, 50-180 $\mu \mathrm{m}$, with FIS (Kawada et al., 2007). The FIS scanned $96 \%$ of the entire sky more than twice in the 16 months of the cryogenic mission phase.

In March, 2010, the AKARI/FIS Bright Source Catalogue V. 1.0 was released to the scientific community. It contains in total 427071 point sources measured at 65,90 , $140,160 \mu \mathrm{m}$. The position accuracy of the FIS sources is $8^{\prime \prime}$, since the source extraction is made with grids of this size.

Effective size of the point spread function of AKARI FIS in FWHM is estimated to be $37 \pm 1^{\prime \prime}, 39 \pm 1^{\prime \prime}, 58 \pm 3^{\prime \prime}$, and $61 \pm 4^{\prime \prime}$ at N60, WIDE-S, WIDE-L, and N160, respectively (Kawada et al., 2007). Errors are not estimated for each individual source, but instead they are, in total, estimated to be $35 \%, 30 \%, 60 \%$, and $60 \%$ at N60, WIDE-S, WIDE-L, and N160, respectively (Yamamura et al., 2009). For the examination of properties of objects on color-color planes, the effect of photometric errors is a matter of concern. In Pollo et al. (2010a), we have examined the effect of photometric errors on the boundary of the star-galaxy classification, and found that this affects the position of the boundary on the color-color plane, but not so significantly that it would change quantitative conclusions. It should be noted that photometric errors do not affect the classification of sources used for the purpose of this work: they are all identified by a direct cross-match with public databases, as explained in the latter part of this section.

In addition to the error estimation, quality flags are attributed to all the detected sources. Their values range from 0 (no detection), through 1 (source not confirmed), 2 (de-

\footnotetext{
${ }^{1}$ Detailed information on the AKARI project, instruments, data and important results can be found via URL: http://www.ir.isas.ac.jp/ASTROF/index-e.html.
} 
tection confirmed, flux not reliable) to 3 (source confirmed and flux reliable). In practice, only flags 1 and 3 dominate. It would seem reasonable to narrow down the analysis only to the flag 3 sources; however, the total number of such sources is small and as a result the statistics becomes poor. For that reason, for the subsequent analysis we decided to use all the detected sources, with due attention. However, we use the safe measurements with flag 3 for a quality check of our results.

Among all the AKARI FIS All-Sky Survey sources, we found 43342 sources with fluxes measured at all four FIR bands.

In order to identify and classify AKARI FIS sources, we searched for counterparts of sources with a complete FIR photometric information in public databases: NED and SIMBAD. The details of this procedure were discussed in Pollo et al. (2010a). We stress that, for the purpose of this work, we use only identifications based on a direct cross-match with public databases. The classification of sources (including stars used in this work) is, in most cases, consistent with the information in the SIMBAD and NED databases. However, since the information given there is not always the most recent, in case of all stars used for this work we searched the available literature and in dubious cases we used the classification given in the most recent publications. The following papers were extremely helpful in this task: Deguchi et al. (1990); Lewis et al. (1995); Young (1995); Josselin et al. (1996); Sloan and Price (1998); Percy et al. (2001); Couch et al. (2003); Jura (2003); Maas et al. (2003); Winters et al. (2003); Whitelock et al. (2006); Szczerba et al. (2007); Mesler and Henson (2008); Ita et al. (2010).

It should be noted here that the results presented in Pollo et al. (2010a) were based on the $\beta$-1 version of the AKARI All-Sky Survey (Yamamura et al., 2008), which contained less sources than were included in the published version of the survey, and preliminary measurements of positions and flux densities. Using the published AKARI FIS Bright Source Catalogue Version 1.0 (hereafter: BSC) data we verified our identifications, which, in most cases, were proved to be correct, i.e. the previously found identification was the closest-found counterpart in the search range of $8^{\prime \prime}$ (smaller than the previously assumed $40^{\prime \prime}$ ), while the median distance between the counterpart and the source was $\sim 4^{\prime \prime}$. Also, most often in the search range of $8^{\prime \prime}$, the chosen counterpart was the only possibility. The risk of missidentification still exists, when such a procedure is applied, but since we are dealing with relatively bright sources, it is small. Also, since our work is mainly statistical, a few possible mis-identifications should not affect the final result.

Using the improved flux densities, as well as only measurements with the best flag 3, from the BSC, we also confirmed the validity of the division lines presented in Pollo et al. (2010a) (Rybka et al., 2011, in preparation).

For the purpose of this work, we use the sources identified in the work of Pollo et al. (2010a), but with confirmed identifications and flux densities from the BSC. Identification of Galactic objects, which are the center of interest of this paper, was confirmed in all cases. However, for some flux densities (mostly at 160, but, in a few cases, $140 \mu \mathrm{m}$ ) zeroes were given. To keep the condition that only sources with complete FIR photometric information are used, we removed these sources with zero flux densities from our sample. This procedure decreased the number of Galactic sources in our sample to $\sim 80 \%$ and is responsible for inconsistency between the numbers presented in this work and the tables presented in Pollo et al. (2010a).

\subsection{Point sources outside the Galactic plane}

The area of low emission of Galactic dust $\left(\leq 10 \mathrm{MJy} \mathrm{sr}^{-1}\right)$ in practice corresponds to the sky area outside the Galactic plane and Magellanic Clouds. In this area, we expected the best efficiency of the identification.

Indeed, for 5176 AKARI sources with the full four-band FIR photometry in the $\beta$-1 version of the All-Sky Survey, detected in this area, we could identify all but 22 sources.

Verified with BSC, all these 5176 sources have a flag 3 measurement in the $\mathrm{S} 90$ band, which implies that they are all confirmed sources and their flux densities at this band should be treated as reliable. However, only 1380 of them have a flag 3 measurement at S140 and 405 at the S65 band. The number of objects having a flag 3, reliable, measurement at all four FIS bands is only 142. For that reason, for the analysis of colors we decided to use all the available data, which, as shown in Pollo et al. (2010a, b), still gives statistically-meaningful results. However, when discussing particular cases, we should be aware of possible biases in color measurements. These issues will be discussed in detail in Rybka et al. (2011, in preparation).

Most of these sources discussed in Pollo et al. (2010a) are nearby galaxies (4272). We also found 349 stars and 48 "other" (extended or line emitters) Galactic sources which mostly are related to stars. As mentioned above, identification of all these 397 Galactic sources has been confirmed in the published BSC. The condition of the full photometric information in FIR was kept in the case of 330 of these sources, i.e. $83 \%$ of them.

The properties of these 330 sources are summarized in Table 1. Most of the Galactic point sources presented there (270 from 330 , i.e. $82 \%$ ) are related either to AGB, or postAGB, stars (this category includes mainly planetary and protoplanetary nebulae, $\mathrm{PNe}$ and $\mathrm{PPNe}$ ). In case of some of them the classification is tentative: they are pulsating red giants possibly being AGBs or close to the AGB phase. Since the primary purpose of this work is to make a first-step estimate of the contribution from the evolved intermediate mass stars to the dust supply and the FIR flux of galaxies, we include also these tentatively-classified objects to our sample: if they are not exactly AGBs, they are pulsating red giants not so far from AGBs in evolutionary stage and physical properties. It should also be noted that, among the remaining sources, there is a certain number of other evolved stars, such as supergiants.

\section{AGB and Post-AGB Stars in the FIR Color- Color Diagrams}

Color-color diagrams of the AGB, and post-AGB, stars from our sample are shown in Fig. 1 and Figs. A.1-A.14. In all these figures, the left panel presents the diagram using all the data, while, in the right panel, only the sources with the secure flag 3 for all the flux densities used in a diagram are shown. Different types of AGB stars, viz., 
Table 1. Classification of 330 Milky Way sources with a complete photometry, located in the sky regions of low $100-\mu \mathrm{m}$ emissivity $\left(I_{100} \leq 10\right.$ MJy $\left.\mathrm{sr}^{-1}\right)$.

\begin{tabular}{llc}
\hline AGB and post-AGB stars & & 270 \\
\hline & Mira-type variables & 89 \\
& AGB stars (without detailed classification) & 66 \\
& Carbon stars & 27 \\
& OH/IR stars & 16 \\
& Post-AGB stars including Planetary Nebulae & 49 \\
\hline probable AGB stars or close & 23 \\
\hline & & 60 \\
\hline & Young stars (including Young Stellar Objects, YSO) & 13 \\
& Faint or poorly known stars & 12 \\
& Main sequence stars & 5 \\
& Double or multiple systems & 13 \\
Supergiants and red giants other than AGBs & 11 \\
IR cirruses & 3 \\
& Other nebulae & 2 \\
Hil regions & 1 \\
\hline
\end{tabular}
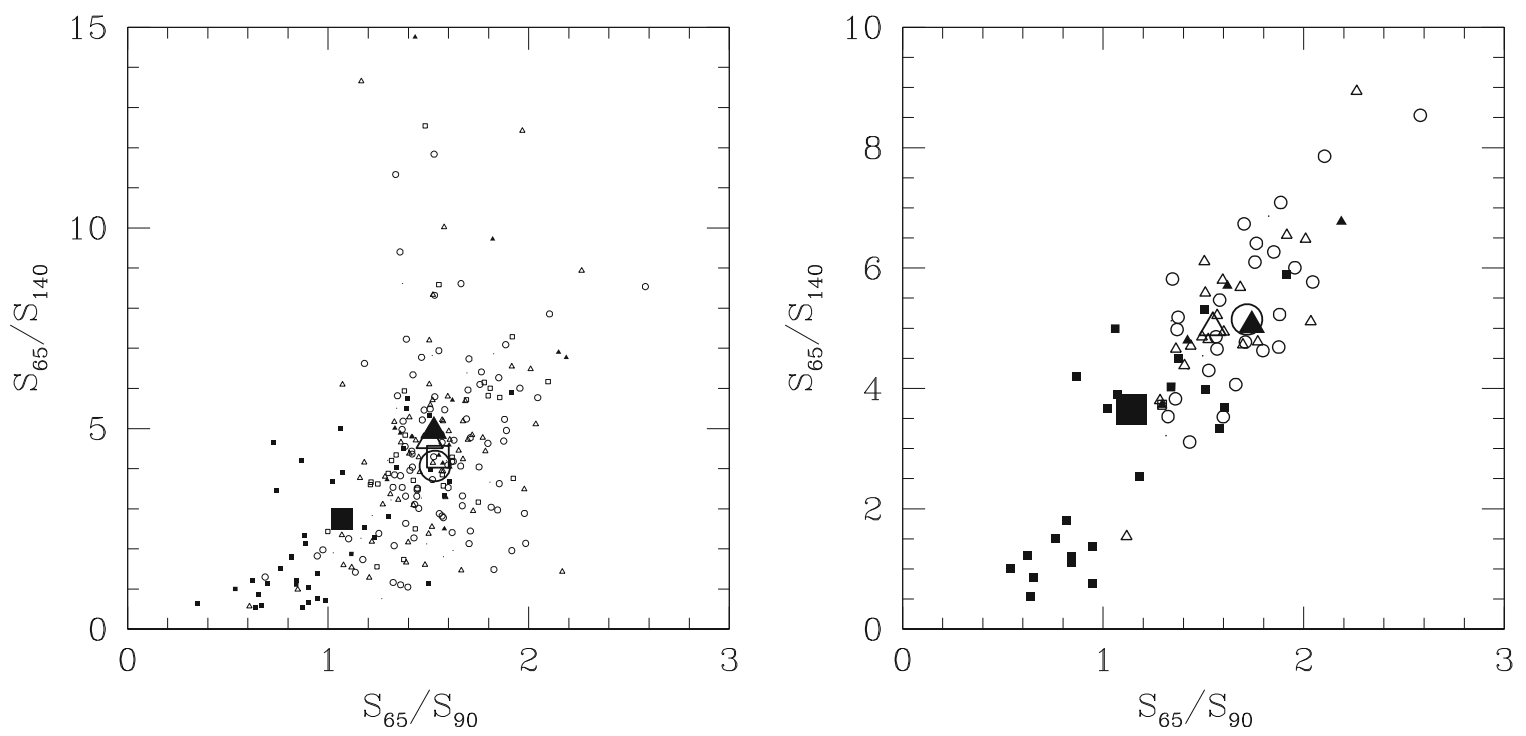

Fig. 1. Positions of different types of AGB stars in the FIR color-color diagram $S_{65} / S_{90}$ vs. $S_{65} / S_{140}$. Mira-type variables are shown as open circles, carbon stars — as open squares, OH/IR stars—as full triangles, post-AGB stars—as full squares, and AGB stars without detailed classification-as open triangles. Individual sources are marked by small symbols, while large symbols denote corresponding median values. In the left panel, all sources are presented. In the right panel, only sources with the highest quality flags 3 are shown (note difference in scale).

carbon stars, $\mathrm{OH} / \mathrm{IR}$ stars, AGB stars without classification, and post-AGB stars, are shown as small symbols, while the corresponding large symbols mark the corresponding median values.

In most of the color-color diagrams, the AGB stars display a significant scatter. This is probably mainly due to the errors in the flux density. This conclusion is supported by the fact that this scatter is much lower in the color using shorter, most secure, wavelengths: $\left(S_{65} / S_{90}\right)$. The scatter is also significantly reduced if we use, in the color-color diagrams, only objects with secure measurements (flag 3).

The median colors of all the sub-types of AGB stars shown in the diagrams are usually very similar. In some of the diagrams (Figs. A.1, A.3, A.5, A.7, A.9, A.10, A.11, A.12, A.14) the OH/IR stars seem to be much bluer than the rest. However, first of all, this is the smallest subcategory of our AGB stars. Second, it should be noted that all these diagrams where the deviation appears make use of the $S_{160}$ flux density, which is not reliable for all but one of these stars (the rest of them received flag 1). Moreover, this single $\mathrm{OH} / \mathrm{IR}$ star with a good $S_{160}$ measurement does not have a reliable measurement of the $S_{140}$ flux density. As a result, only one OH/IR star is shown in only some of the "high quality" diagrams using the $S_{160}$ flux density, and, of course, without a corresponding median. However, in the "high quality" diagrams using only shorter wavelengths these stars do not seem to differ from the rest of the AGB stars. For that reason, we do not regard this deviation as being significant-it is probably due to the combined effect of small statistics and large errors.

The same problem of a small number of high-quality measurements in $S_{160}$ applies to carbon stars which are the 


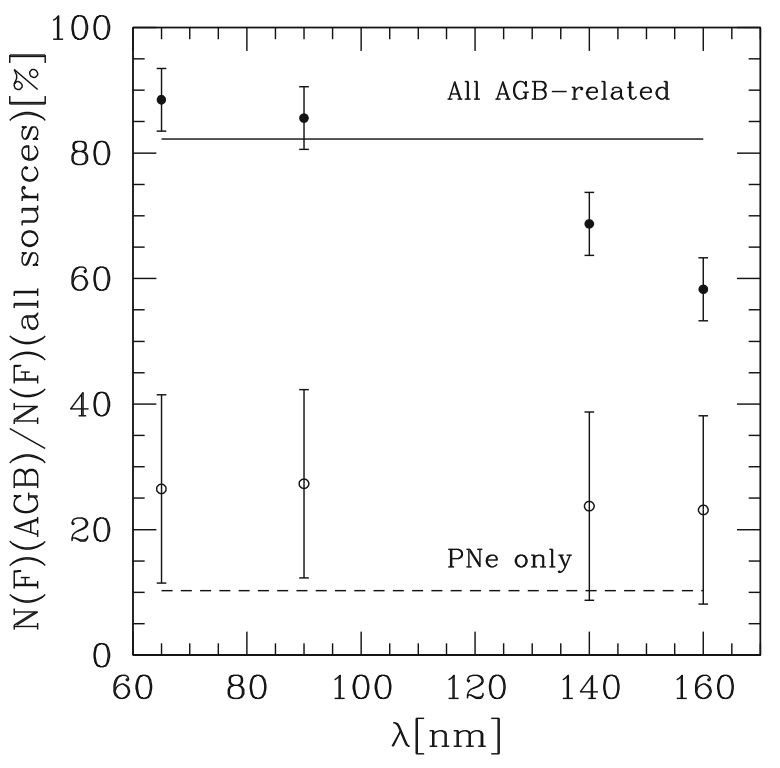

Fig. 2. Contribution from AGB stars to the total number of point sources detected in FIR (solid horizontal line for all sources and dashed horizontal line for planetary nebulae) and to the total FIR flux of the Milky Way outside of the Galactic plane, coming from point sources (full circles for all sources and empty circles for planetary nebulae only) in different FIR wavelengths from 65 to $160 \mu \mathrm{m}$. Indicated errors are rms errors, related to the number statistics of sources.

second-smallest subcategory of our sample of AGB stars. Consequently, their corresponding medians are also not shown in the "high quality" versions of the diagrams. However, in the remaining diagrams, they also seem to be placed very similarly to other AGB stars.

At the same time, the only group which appears to be separated in most of the diagrams are post-AGB stars, or, more precisely, a subsample of post-AGB stars. As shown in the $S_{65} / S_{90}$ vs. $S_{65} / S_{140}$ diagram, presented in Fig. 1, these objects are significantly redder and this effect remains visible also in the diagram using only the secure flag 3 . Especially in the right panel of Fig. 1, which presents the diagram using only "secure" measurements, it is seen that some post-AGB objects (all of them are planetary nebulae) form a clearly-separated "cloud" in the red part of the diagram. Another group of post-AGB objects is mixed with the main AGB cloud, but also seems to remain in its redder part. Consequently, the median value for the post-AGB stars is shifted closer to the main cloud, but the difference with respect to the AGB stars is still evident. The same effect is visible in practically all the other diagrams, presented in Figs. A.1-A.14.

As an attempt to interpret this behavior, we present the identifications of these "red" and "blue" clouds of postAGB objects, respectively, in Tables 2 and 3. All of these sources are well-known PNe, with the exception of one PPN. In the first and second column of Tables 2 and 3, we give the most commonly-used names of these nebulae, while, in the third column, we put the comments about the morphologies and estimation of their kinematic ages, taken from the literature. Since the estimation of the ages of planetary nebulae depends on the estimation of their distances, very often highly uncertain, there are many contradictory estimations found in the literature and the numbers given in the tables should be treated as indicative. The evolutionary stage of planetary nebulae does not depend only on their age, but also on other factors, such as the mass of the exciting star (see, e.g. Blöcker, 1995). Given a small number of objects, we do not attempt to formulate any strong conclusions about the relation between the properties of these objects and their position in the FIR color-color diagrams. We observe, however, that most of the members of the bluer cloud of post-AGB stars are young PNe, and their morphological structure is usually not very well developed. The PPN Frosty Leo also belongs to this group. Even older PNe in this group, like the Saturn Nebula, are not strongly evolved yet, either. The remarkable difference is a particular born-again PN Abell 78.

In contrast, the members of the red cloud of post-AGB objects usually have well-developed morphological structures. They are very often bipolar, with strong asymmetrical features. Their estimated ages are usually older.

Given the above observations, we can formulate the careful speculation that PNe move towards the redder part of the FIR color-color diagram (i.e. become more luminous in the $S_{140}$ and $S_{160}$ bands) as a result of their evolution. This is related not simply to their age, but rather to the evolutionary status and stage of development of the nebula. If so, this reddening may be simply interpreted as a result of the cooling of grains (a similar interpretation is given by Phillips and Marquez-Lugo, 2011, who analysed a much larger sample of planetary nebulae observed by AKARI). It is also possible that the very often seen complex bipolar structure of these redder nebulae is related to their increased luminosity in the longer IR wavelengths, and that both these features have a common origin. This issue undoubtedly will be a subject of further studies.

The consequences of this effect for the observed flux of the outer part of the Milky Way are discussed in the next section.

\section{FIR Flux from AGB and Post-AGB Stars- Contribution to the Total FIR Flux Related to Point Sources outside the Galactic Plane}

As seen from Table 1, AGB, or plausible AGB, stars are more than $80 \%$ of Galactic FIR point sources detected outside the Galactic plane. Planetary nebulae are $10 \%$ of these sources. These percentages are also presented in Fig. 2 as vertical lines.

At the same time, as shown in Fig. 2, the contribution from AGB stars to the total FIR flux from Milky Way sources located outside the Galactic plane corresponds to their number percentage but changes with wavelength. It is high in all the range between 65 and $160 \mu \mathrm{m}$, above $85 \%$ at $65 \mu \mathrm{m}$, and dropping above $100 \mu \mathrm{m}$, but at $160 \mu \mathrm{m}$ still remaining at a level of more than $60 \%$.

More than $20 \%$ of this contribution to the FIR flux comes from post-AGB stars (mainly planetary nebulae), while, in number, they only account for $10 \%$ of the sources. Their contribution becomes more significant at longer wavelengths, since a significant fraction of them does not become fainter at wavelengths longer than $100 \mu \mathrm{m}$, contrary to the other stars. A subsample of post-AGB stars which 
Table 2. Identifications and properties of the separated red cloud of post-AGB stars selected in the high-quality color-color diagram $S_{65} / S_{90}$ vs. $S_{65} / S_{160}$, presented in the right panel of Fig. 1.

\begin{tabular}{|c|c|c|}
\hline Name & Other common name(s) & Relevant information \\
\hline \multirow[t]{2}{*}{ M 1-7 } & IRAS $06342+2403$ & compact, bright, $t \sim 6000 \mathrm{yrs}$ \\
\hline & 2MASX J06372096+2400365 & (Sabbadin et al., 1984) \\
\hline NGC 2371-2 & & dual lobed, bipolar, evolved \\
\hline \multirow[t]{2}{*}{ NGC 2392} & Eskimo Nebula, & two-shell, bipolar \\
\hline & Clownface Nebula & $t \sim 1050 \mathrm{yrs}\left(\mathrm{O}^{\prime}\right.$ Dell et al., 2002) \\
\hline NGC 3195 & PN G296.6-20.0 & large, evolved \\
\hline IC 4406 & Retina Nebula & bipolar \\
\hline ESO 229-6 & Sp3; PN G342.5-14.3 & $t \sim 7000$ yrs (Gauba et al., 2001) \\
\hline M 57 & Ring Nebula; NGC 6720 & $t \sim 7000$ yrs (O’Dell et al., 2007, 2009) \\
\hline ESO 524-06 & HaWe 12 & possible planetary nebula \\
\hline NGC 6905 & The Blue Flash Nebula & bipolar \\
\hline \multirow[t]{5}{*}{ M 2-9 } & V0651 Mon; Butterfly Nebula & bipolar \\
\hline & “Twin Jet” Nebula & $t \sim 2500 \mathrm{yrs}($ Corradi et al., 2011) \\
\hline & & possibly a double system of WD and AGB, or post-AGB \\
\hline & & 1200 yrs after a symbiotic nova eruption (Livio and Soker, 2001) \\
\hline & & or with a dusty disk (e.g. Lykou et al., 2011) \\
\hline
\end{tabular}

Table 3. Identifications and properties of the bluer cloud of post-AGB stars, mixed with the AGB stars, selected in the high-quality color-color diagram $S_{65} / S_{90}$ vs. $S_{65} / S_{160}$, presented in the right panel of Fig. 1.

\begin{tabular}{|c|c|c|}
\hline Name & Other common name(s) & Relevant information \\
\hline \multirow[t]{2}{*}{ IC 2149} & & bipolar (in the process of forming?) \\
\hline & & (Vázquez et al., 2002) \\
\hline \multirow[t]{2}{*}{ NGC 2818} & & in front of an open cluster C 0914-364, bipolar \\
\hline & & age $600-1000$ yrs? \\
\hline \multicolumn{2}{|l|}{ Frosty Leonis Nebula } & protoplanetary nebula, bipolar \\
\hline \multirow[t]{2}{*}{ NGC 3242} & Ghost of Jupiter & $t \sim 2800 \mathrm{yrs}$ \\
\hline & & (Steffen et al., 2008) \\
\hline \multirow[t]{2}{*}{ NGC 6210} & & $t \sim 2000 \mathrm{yrs}$ \\
\hline & & (Pottasch et al., 2009) \\
\hline \multirow[t]{2}{*}{ NGC 6826} & Blinking Nebula & almost circular, early evolutionary status \\
\hline & & $t \sim 5000$ yrs (Guerrero et al., 1998) \\
\hline \multirow[t]{2}{*}{ NGC 7009} & Saturn Nebula & $t \sim 7000$ yrs (but not much evolved) \\
\hline & & (Sabbadin et al., 2004) \\
\hline \multirow[t]{2}{*}{ Abell 78} & PN G081.2-14.9 & hydrogen-defficient "born-again PNe" after a He flash \\
\hline & & (Borkowski et al., 1993) \\
\hline \multirow[t]{2}{*}{ NGC 7662} & Blue Snowball Nebula & triple shell; ellipsoidal \\
\hline & Caldwell 22 & $t \sim 1050$ yrs (Guerrero et al., 2004) \\
\hline
\end{tabular}

form a separate redder group on the color-color diagrams is responsible for this effect.

\section{Summary}

We conclude that Galactic objects luminous in the FIR outside the Galactic plane in more than $80 \%$ of cases are red giants, mainly AGB, and post-AGB, stars. They dominate not only in number, but also their contribution to the FIR flux associated with point sources in the outer part of the Galaxy is dominant. While it has already been established that a significant mass fraction of Galactic dust is related to AGB stars, now we show that they also contribute significantly to the FIR flux of the outer parts of our Galaxy, thanks to the new data from AKARI.

This issue needs to be studied further but we may expect a similar behavior for other evolved Milky Way-type galaxies. Further exploration of AKARI All Sky Survey data should provide a more precise estimation of the role of the AGB component in the FIR spectral-energy distribution of the Milky Way and other galaxies. In particular, our measurement implies that the contribution from stars in late phases of evolution should probably not be neglected when modeling the FIR emission of Milky Way-type galaxies.

Additionally, we point out that the FIR color-color diagrams only provide a possibility for the successful selection of a sample of post-AGB stars, in particular of planetary nebulae.

Acknowledgments. This work is based on observations with AKARI, a JAXA project with the participation of ESA. This research has made use of the NASA/IPAC Extragalactic Database (NED) which is operated by the Jet Propulsion Laboratory, California Institute of Technology, under contract with the National Aeronautics and Space Administration, and the SIMBAD database, operated at CDS, Strasbourg, France. We deeply thank Issei Yamamura for his huge effort of checking the reliability of our stellar identifications and their classification. AP was fi- 
nanced by the research grant of the Polish Ministry of Science PBZ/MNiSW/07/2006/34A. AP and PR were financed by the research grant of the Polish Ministry of Science N N203 512938. TTT has been supported by Program for Improvement of Research Environment for Young Researchers from Special Coordination Funds for Promoting Science and Technology, and the Grant-inAid for the Scientific Research Fund (20740105, 23340046) commissioned by the Ministry of Education, Culture, Sports, Science and Technology (MEXT) of Japan. TTT and PR have been partially supported from the Grand-in-Aid for the Global COE Program "Quest for Fundamental Principles in the Universe: from Particles to the Solar System and the Cosmos" from the MEXT.

\section{References}

Asano, R. S., T. T. Takeuchi, H. Hirashita, and A. K. Inoue, Dust formation history of galaxies: dependence on the sources of dust production, Astron. Astrophys., submitted.

Blöcker, T., Stellar evolution of low and intermediate-mass stars. I. Mass loss on the AGB and its consequences for stellar evolution, Astron. Astrophys., 297, 727, 1995.

Borkowski, K. J., J. P. Harrington, Z. Tsvetanov, and R. E. S. Clegg, HST imaging of hydrogen-poor ejecta in Abell 30 and Abell 78-Windblown cometary structures, Astrophys. J., 415, L47-L50, 1993.

Buat, V., T. T. Takeuchi, J. Iglesias-Páramo et al., The local Universe as seen in the far-infrared and far-ultraviolet: A global point of view of the local recent star formation, Astrophys. J. Suppl., 173, 404-414, 2007a.

Buat, V., D. Marcillac, D. Burgarella et al., The ultraviolet properties of luminous infrared galaxies at $z \sim 0.7$. Is there any evolution in their dust attenuation?, Astron. Astrophys., 469, 19-25, $2007 \mathrm{~b}$.

Buat, V., S. Boissier, D. Burgarella et al., Star formation history of galaxies from $z=0$ to $z=0.7$. A backward approach to the evolution of starforming galaxies, Astron. Astrophys., 483, 107-119, 2008.

Busso, M., L. Origlia, M. Marengo, P. Persi, M. Ferrari-Toniolo, G. Silvestro, L. Corcione, M. Tapia, and J. Bohigas, Mid-infrared imaging of AGB star envelopes. I. Colors, classification and mass-loss estimates, Astron. Astrophys., 311, 253-263, 1996.

Caputi, K., G. Lagache, L. Yan et al., The infrared luminosity function of galaxies at redshifts $z=1$ and $z \sim 2$ in the GOODS fields, Astrophys. J., 660, 97-116, 2007.

Clayton, D. D., E. A.-N. Deneault, and B. S. Meyer, Condensation of carbon in radioactive supernova gas, Astrophys. J., 562, 480-493, 2001.

Corradi, R. L. M., B. Balick, and M. Santander-Garcia, The evolution of M 2-9 from 2000 to 2010, Astron. Astrophys., 529, A43, 2011.

Couch, P. A., T. Lloyd Evans, and P. J. Sarre, The cool circumstellar environment of IRAS 08182-6000: radiative transfer modeling of TiO absorption bands, Mon. Not. R. Astron. Soc., 346, 153-162, 2003.

Cox, N. L. J., D. A. Garcia-Hernandez, P. Garcia-Lario, and A. Manchado, Far-infrared imaging of post-AGB stars and (proto)-planetary nebulae with the AKARI Far-Infrared Surveyor, Astron. J., arXiv:1101.3450, 2011 (accepted).

Deguchi, S., Y. Nakada, and R. Sahai, SiO and CO emission from carbon stars with silicate features and southern IRAS sources, Astron. Astrophys., 230, 339-354, 1990.

Dwek, E., The evolution of the elemental abundances in the gas and dust phases of the galaxy, Astrophys. J., 501, 643, 1998.

Gauba, G., M. Parthasarathy, Y. Nakada, and T. Fujii, UV (IUE) spectra of the central stars of high latitude planetary nebulae $\mathrm{Hb} 7$ and $\mathrm{Sp} 3$, Astron. Astrophys., 373, 572, 2001

Guerrero, M. A., E. Villaver, and A. Manchado, On the kinematics of multiple-shell planetary nebulae. I. Data and expansion velocities, Astrophys. J., 507, 889-908, 1998.

Guerrero, M. A., E. G. Jaxon, and Y.-H. Chu, Physical structure of planetary nebulae. II. NGC 7662, Astrophys. J., 128, 1705, 2004.

Hacking, P. et al., The brightest high-latitude 12-micron IRAS sources, Publ. Astron. Soc. Pac., 97, 616-633, 1985.

Ita, Y. et al., AKARI's infrared view on nearby stars. Using AKARI infrared camera all-sky survey, 2MASS, and Hipparcos catalogs, Astron. Astrophys., 514(A2), 2010.

Josselin, E., C. Loup, A. Omont, C. Barnbaum, and L.-A. Nyman, Infrared giants vs. supergiants. I. Far infrared-to- $\mathrm{CO}(1-0)$ intensity ratio, Astron. Astrophys., 315, L23-L26, 1996.

Jura, M., A flared, orbiting, dusty disk around HD 233517, Astron. J., 582, 1032-1035, 2003.

Kawada, M. et al., The Far-Infrared Surveyor (FIS) for AKARI, Publ.
Astron. Soc. Jpn., 59, 389-400, 2007.

Kelson, D. D. and B. P. Holden, The mid-infrared luminosities of normal galaxies over cosmic time, Astrophys. J., 713, L28-L32, 2010.

Kozasa, T. and H. Hasegawa, Grain formation through nucleation process in astrophysical environments. II-Nucleation and grain growth accompanied by chemical reaction-, Prog. Theor. Phys., 77, 1402-1410, 1987.

Kozasa, T., H. Hasegawa, and K. Nomoto, Formation of dust grains in the ejecta of SN 1987A. II, Astron. Astrophys., 249, 474-482, 1991.

Le Floc'h, E., C. Papovich, and H. Dole, Infrared luminosity functions from the Chandra deep field-south: The Spitzer view on the history of dusty star formation at $0<z<1$, Astrophys. J., 632, 169-190, 2005.

Lewis, B. M., P. David, and A. M. Le Squeren, Mainline OH detection rates from blue circumstellar shells, Astron. Astrophys. Suppl., 111, 237, 1995.

Livio, M. and N. Soker, The "Twin Jet" planetary nebula M2-9, Astrophys. J., 552, 685-691, 2001.

Lykou, F., O. Chesneau, A. A. Zijlstra, A. Castro-Carrizo, E. Lagadec, B. Balick, and N. Smith, A disc inside the bipolar planetary nebula M2-9, Astron. Astrophys., 527, A105, 2011.

Maas, T., H. Van Winckel, T. Lloyd Evans, L.-A. Nyman, D. Kilkenny, P. Martinez, F. Marang, and F. van Wyk, IRAS 08544-4431: A new post-AGB star in a binary system surrounded by a dusty disc, Astron. Astrophys., 405, 271-283, 2003.

Mesler, R. A. and G. D. Henson, Luminosity variations in post-AGB stars, J. Southeastern Assoc. Res. Astron., 2, 10-13, 2008.

Murakami, H. et al., The infrared astronomical mission AKARI, Publ. Astron. Soc. Jpn., 59, 369-376, 2007.

Neugebauer, G. et al., The Infrared Astronomical Satellite (IRAS) mission, Astrophys. J., 278, L1-L4, 1984.

Nozawa, T., T. Kozasa, H. Umeda, K. Maeda, and K. Nomoto, Dust in the early Universe: Dust formation in the ejecta of population III Supernovae, Astrophys. J., 598, 785-803, 2003.

Nozawa, T., T. Kozasa, A. Habe, E. Dwek, H. Umeda, N. Tominaga, K. Maeda, and K. Nomoto, Evolution of dust in primordial supernova remnants: Can dust grains formed in the ejecta survive and be injected into the early interstellar medium?, Astrophys. J., 666, 955-966, 2007.

Nozawa, T. et al., Early formation of dust in the ejecta of type Ib SN 2006jc and temperature and mass of the dust, Astrophys. J., 684, 1343-1350, 2008.

Nozawa, T., T. Kozasa, N. Tominaga, K. Maeda, H. Umeda, K. Nomoto, and O. Krause, Formation and evolution of dust in type IIb supernovae with application to the Cassiopeia A supernova remnant, Astrophys. J., 713, 356-373, 2010.

O’Dell, C. R., B. Balick, A. R. Hajian, W. J. Henney, and A. Burkert, Knots in nearby planetary nebulae, Astron. J., 123, 3329-3347, 2002.

O'Dell, C. R., F. Sabbadin, and W. J. Henney, The three-dimensional ionization structure and evolution of NGC 6720, the ring nebula, Astron. J., 134, 1679-1692, 2007.

O’Dell, C. R., W. J. Henney, and F. Sabbadin, Tangential motions and spectroscopy within NGC 6720, the ring nebula, Astron. J., 137, 38153825, 2009.

Onaka, T. et al., The Infrared Camera (IRC) for AKARI-Design and imaging performance, Publ. Astron. Soc. Jpn., 59, S401-S410, 2007.

Percy, J. R., J. B. Wilson, and G. W. Henry, Long-term VRI photometry of small-amplitude red variables. I. Light curves and periods, Publ. Astron. Soc. Pac., 113, 989-996, 2001.

Phillips, J. P. and R. A. Marquez-Lugo, Mid and far infrared photometry of galactic planetary nebulae with the AKARI all sky survey, Revista Mexicana de Astronomía y Astrofísica, arXiv:1102.0526, 2011 (submitted).

Pipino, A., X. L. Fan, F. Matteucci, F. Calura, L. Silva, G. Granato, and R. Maiolino, The chemical evolution of elliptical galaxies with stellar and QSO dust production, Astron. Astrophys., 525, A61, 2011.

Pollo, A., P. Rybka, and T. T. Takeuchi, Star-galaxy separation by farinfrared color-color diagrams for the AKARI FIS all-sky survey (bright source catalog version $\beta$-1), Astron. Astrophys., 514, 3-21, 2010a.

Pollo, A., P. Rybka, and T. T. Takeuchi, AKARI all-sky surveyContribution from AGB stars to the far infrared flux of Milky Way, in Why Galaxies Care about AGB Stars II: Shining Examples and Common Inhabitants, edited by Kerschbaum, F., T. Lebzelter, and B. Wing. Astron. Soc. Pac. Conf. Ser., San Francisco, 2010b (in press).

Pottasch, S. R., J. Bernard-Salas, and T. R. Roeling, Abundances in the planetary nebula NGC 6210, Astron. Astrophys., 499, 249, 2009.

Reddy, N. A., C. C. Steidel, M. Pettini et al., Multiwavelength constraints on the cosmic star formation history from spectroscopy: the rest-frame ultraviolet, $\mathrm{H} \alpha$, and infrared luminosity functions at redshifts $1.9<z<$ 
3.4, Astrophys. J. Suppl. Ser., 175, 48-85, 2008.

Rho, J., T. Kozasa, W. T. Reach, J. D. Smith, L. Rudnick, T. DeLaney, J. A. Ennis, H. Gomez, and A. Tappe, Freshly formed dust in the Cassiopeia A supernova remnant as revealed by the Spitzer space telescope, Astrophys. J., 673, 271-282, 2008

Rho, J., W. T. Reach, A. Tappe, U. Hwang, J. D. Slavin, T. Kozasa, and L. Dunne, Spitzer observations of the young core-collapse supernova remnant 1E0102-72.3: Infrared ejecta emission and dust formation, Astrophys. J., 700, 579-596, 2009.

Rybka, P. et al., 2011 (in preparation).

Sabbadin, F., S. Ortolani, and R. Falomo, The planetary nebula M 1-7, Astron. Astrophys., 137, 177-184, 1984.

Sabbadin, F., M. Turatto, F. Cappellaro, S. Benetti, and R. Ragazzoni, The 3-D ionization structure and evolution of NGC 7009 (Saturn Nebula). Astron. Astrophys., 416, 955-981, 2004.

Schlegel, D. J., D. P. Finkbeiner, and M. Davis, Maps of dust infrared emission for use in estimation of reddening and cosmic microwave background radiation foregrounds, Astrophys. J., 500, 525, 1998.

Sloan, G. C. and S. D. Price, The infrared spectral classification of oxygenrich dust shells, Astrophys. J. Suppl. Ser., 119, 141-158, 1998.

Steffen, M., D. Schönberner, and A. Warmuth, The evolution of planetary nebulae. V. The diffuse X-ray emission, Astron. Astrophys., 489, 173, 2008.

Szczerba, R., N. Siódmiak, G. Stasińska, and J. Borkowski, An evolutionary catalogue of galactic post-AGB and related objects, Astron. Astrophys., 469, 799-806, 2007.

Takeuchi, T. T., V. Buat, and D. Burgarella, The evolution of the ultraviolet and infrared luminosity densities in the universe at $0<z<1$, Astron. Astrophys., 440, L17-L20, 2005.

Takeuchi, T. T., R. S. Asano, T. T. Ishii, T. Nozawa, H. Hirashita, and T. Kozasa, Dust SED model of very young galaxies with AGB dust, in Why Galaxies Care About AGB Stars II: Shining Examples and Common Inhabitants, edited by Kerschbaum, F., T. Lebzelter, and B. Wing, Astron. Soc. Pac. Conf. Ser., San Francisco, 2010 (in press).
Thronson, H. A., Jr., W. B. Latter, J. H. Black, J. Bally, and P. Hacking, Properties of evolved mass-losing stars in the Milky Way and variations in the interstellar dust composition, Astrophys. J., 322, 770-786, 1987. Valiante, R., R. Schneider, S. Bianchi, and A. C. Andersen, Stellar sources of dust in the high-redshift Universe, Mon. Not. R. Astron. Soc., 397, 1661-1671, 2009.

van der Veen, W. E. C. J. and H. J. Habing, The IRAS two-colour diagram as a tool for studying late stages of stellar evolution, Astron. Astrophys., 194, 125-134, 1988.

Vázquez, R., L. F. Miranda, J. M. Torrelles, L. Olguín, G. Benítez, L. F. Rodríguez, and J. A. López, Multiwavelength observations of the peculiar planetary nebula IC 2149, Astrophys. J., 576, 860, 2002.

Walker, H. J. and M. Cohen, The classification of stars from IRAS colors, Astron. J., 95, 1801-1816, 1988.

Walker, H. J., K. Volk, R. J. Wainscoat, D. E. Schwartz, and M. A. Cohen, A practical approach to classification of IRAS sources using infrared colors alone, Astron. J., 98, 2163-2181, 1989.

Whitelock, P. A., M. W. Feast, F. Marang, and M. A. T. Groenewegen, Near-infrared photometry of carbon stars, Mon. Not. R. Astron. Soc., 369, 751-782, 2006.

Winters, J. M., T. Le Bertre, K. S. Jeong, L.-A. Nyman, and N. Epchtein, Mass-loss from dusty, low outflow-velocity AGB stars. I. Wind structure and mass-loss rates, Astron. Astrophys., 409, 715-735, 2003.

Yamamura, I., Y. Fukuda, and S. Makiuti, AKARI/FIS all-sky survey bright source catalogue version $\beta$-1 release note (rev 1), 2008.

Yamamura, I., S. Makiuti, N. Ikeda et al., Release of the AKARI-FIS bright source catalogue $\beta-1$, in AKARI, a Light to Illuminate the Misty Universe, edited by Onaka, T., G. White, T. Nakagawa, and I. Yamamura, pp. 3, Astron. Soc. Pac. Conf. Ser., 418, San Francisco, 2009.

Young, K., A CO(3-2) survey of nearby Mira variables, Astrophys. J., 445, 842-888, 1995.

A. Pollo (e-mail: apollo@fuw.edu.pl), T. T. Takeuchi, and P. Rybka 


\section{Appendix A. Remaining Color-Color Diagrams}
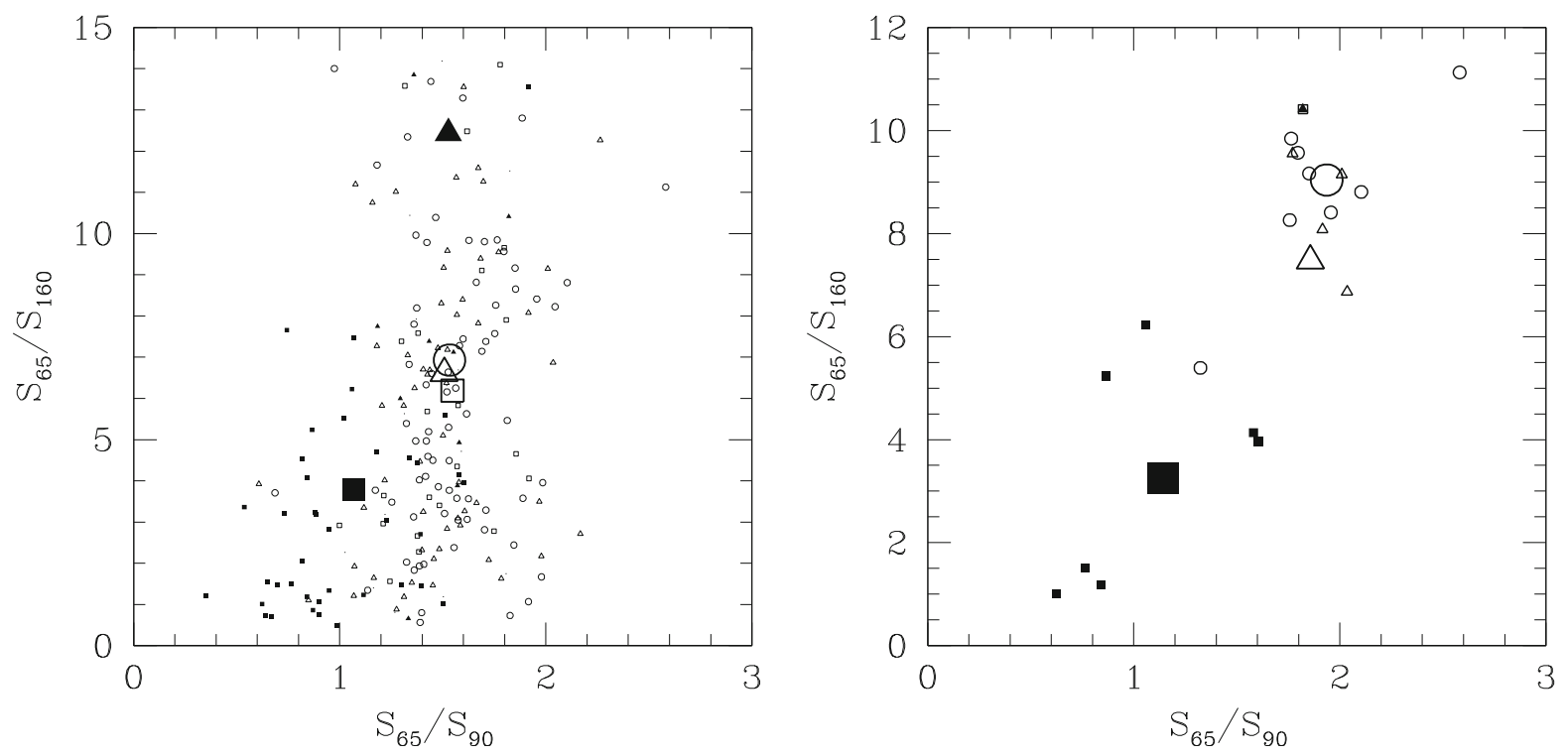

Fig. A.1. Positions of different types of AGB stars in the FIR color-color diagram $S_{65} / S_{90}$ vs. $S_{65} / S_{160}$. All symbols as in Fig. 1 . In the left panel, all sources are presented. In the right panel, only sources with quality flags 3 are included (note difference in scale). Since the OH/IR stars, the least numerous in our classification in general, do not have enough number of reliable measurements in the $S_{160}$ band, their corresponding median color does not appear in the right panel in the plots using colors based on the $S_{160}$ flux.
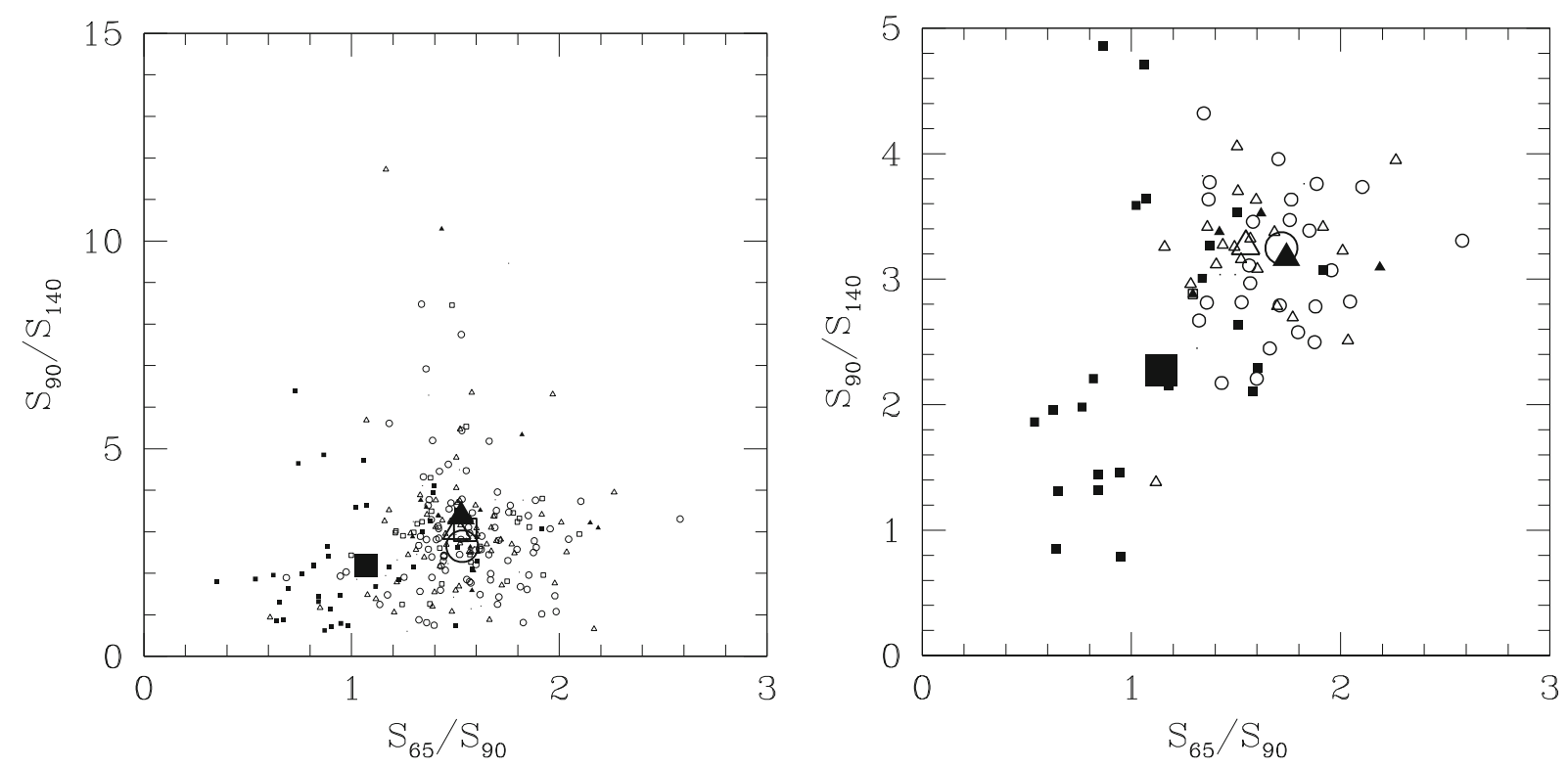

Fig. A.2. Positions of different types of AGB stars in the FIR color-color diagram $S_{65} / S_{90}$ vs. $S_{90} / S_{140}$. All symbols as in Fig. 1 . In the left panel, all sources are presented. In the right panel, only sources with quality flags 3 are included (note difference in scale). 

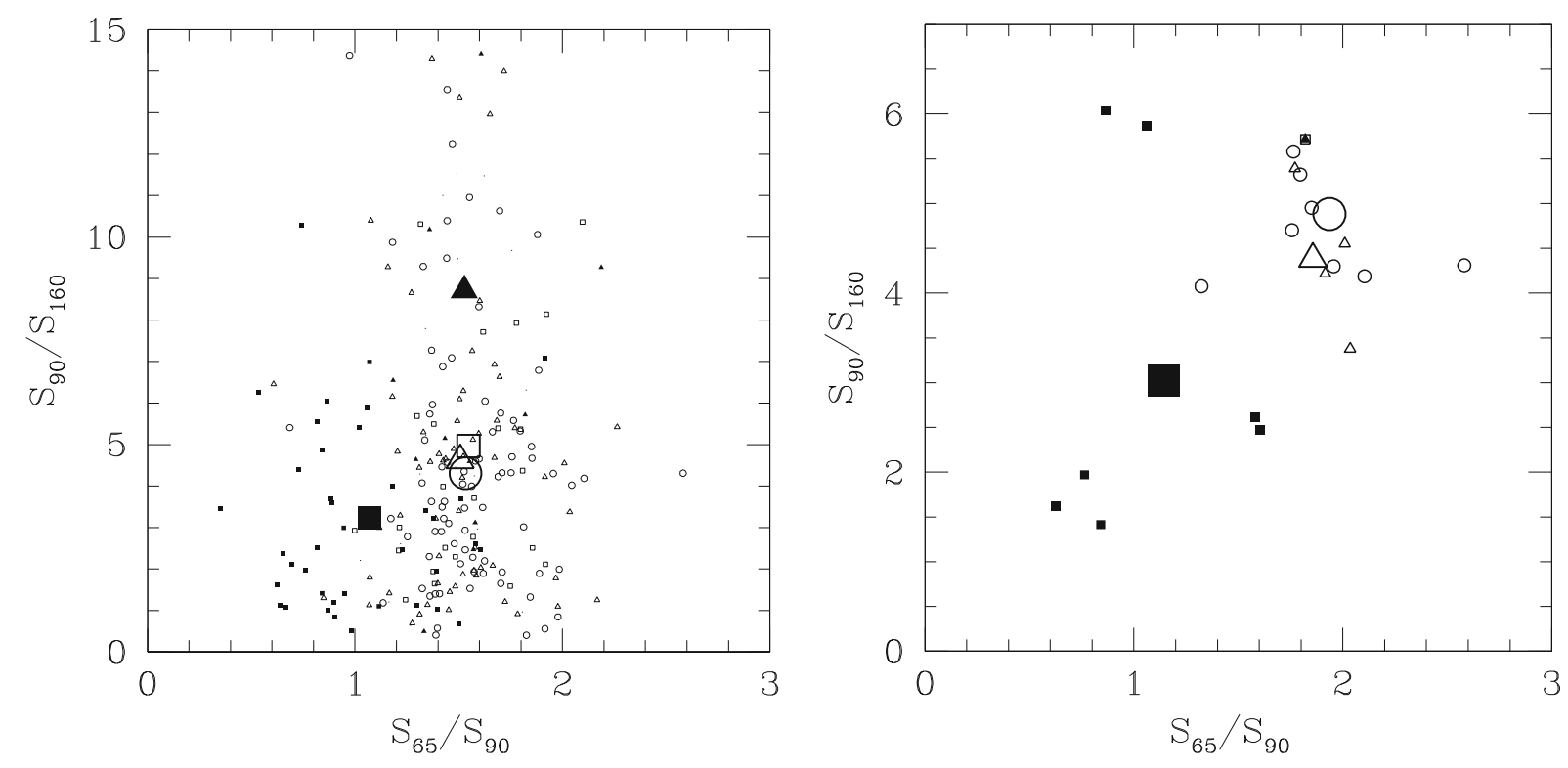

Fig. A.3. Positions of different types of AGB stars in the FIR color-color diagram $S_{65} / S_{90}$ vs. $S_{90} / S_{160}$. All symbols as in Fig. 1 . In the left panel, all sources are presented. In the right panel, only sources with quality flags 3 are included (note difference in scale).
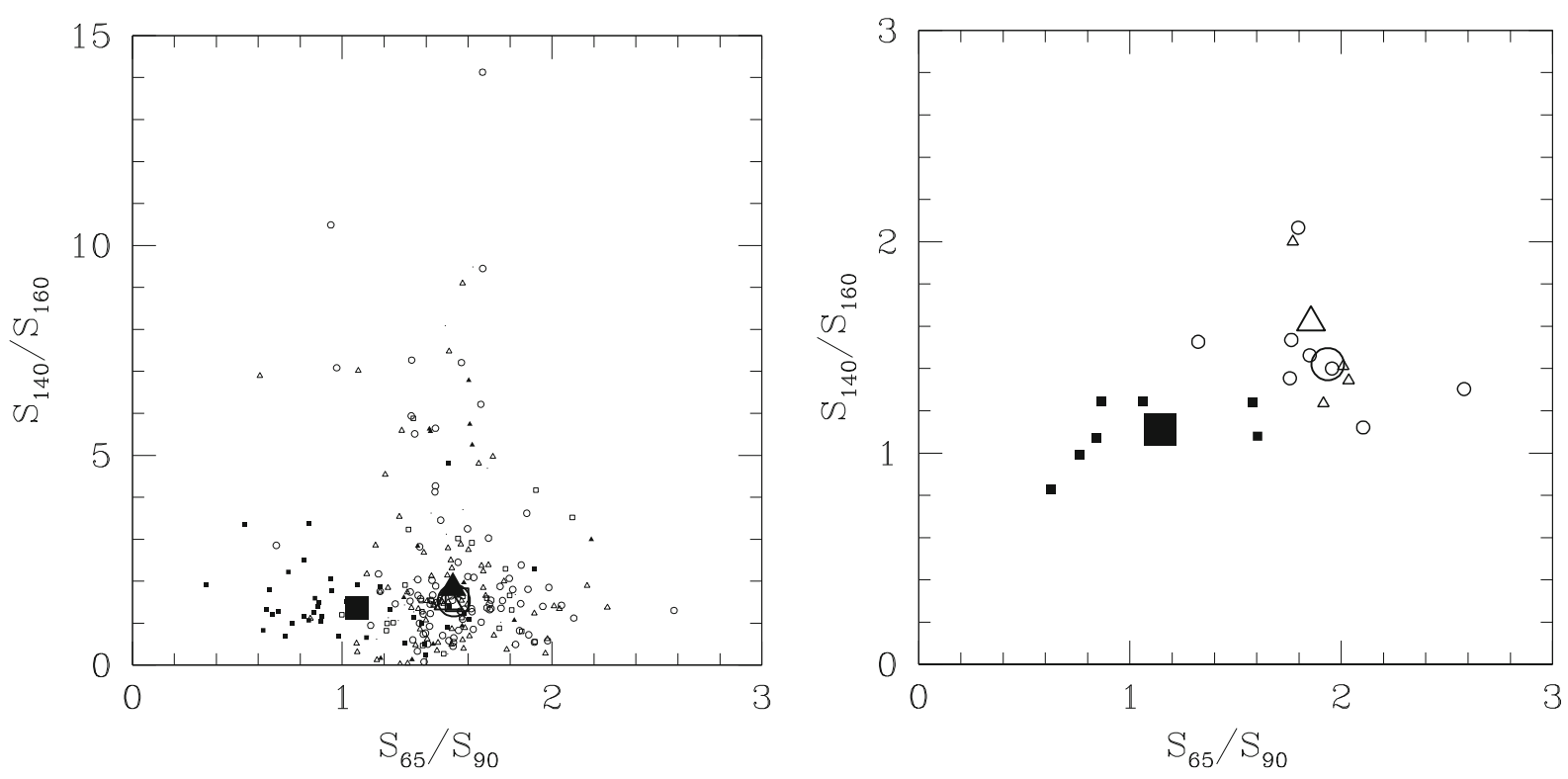

Fig. A.4. Positions of different types of AGB stars in the FIR color-color diagram $S_{65} / S_{90}$ vs. $S_{140} / S_{160}$. All symbols as in Fig. 1 . In the left panel, all sources are presented. In the right panel, only sources with quality flags 3 are included (note difference in scale). 

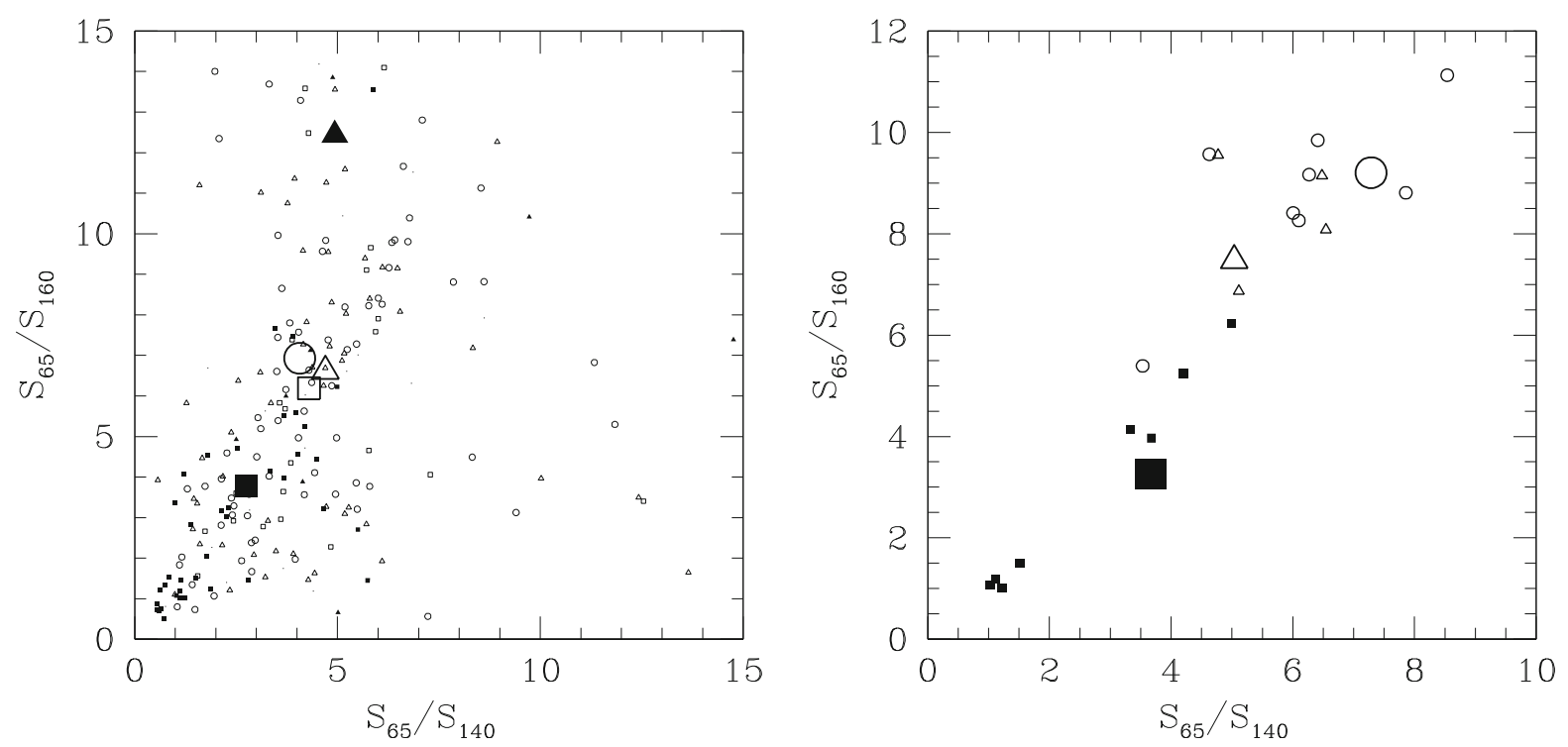

Fig. A.5. Positions of different types of AGB stars in the FIR color-color diagram $S_{65} / S_{140}$ vs. $S_{65} / S_{160}$. All symbols as in Fig. 1. In the left panel, all sources are presented. In the right panel, only sources with quality flags 3 are included (note difference in scale).
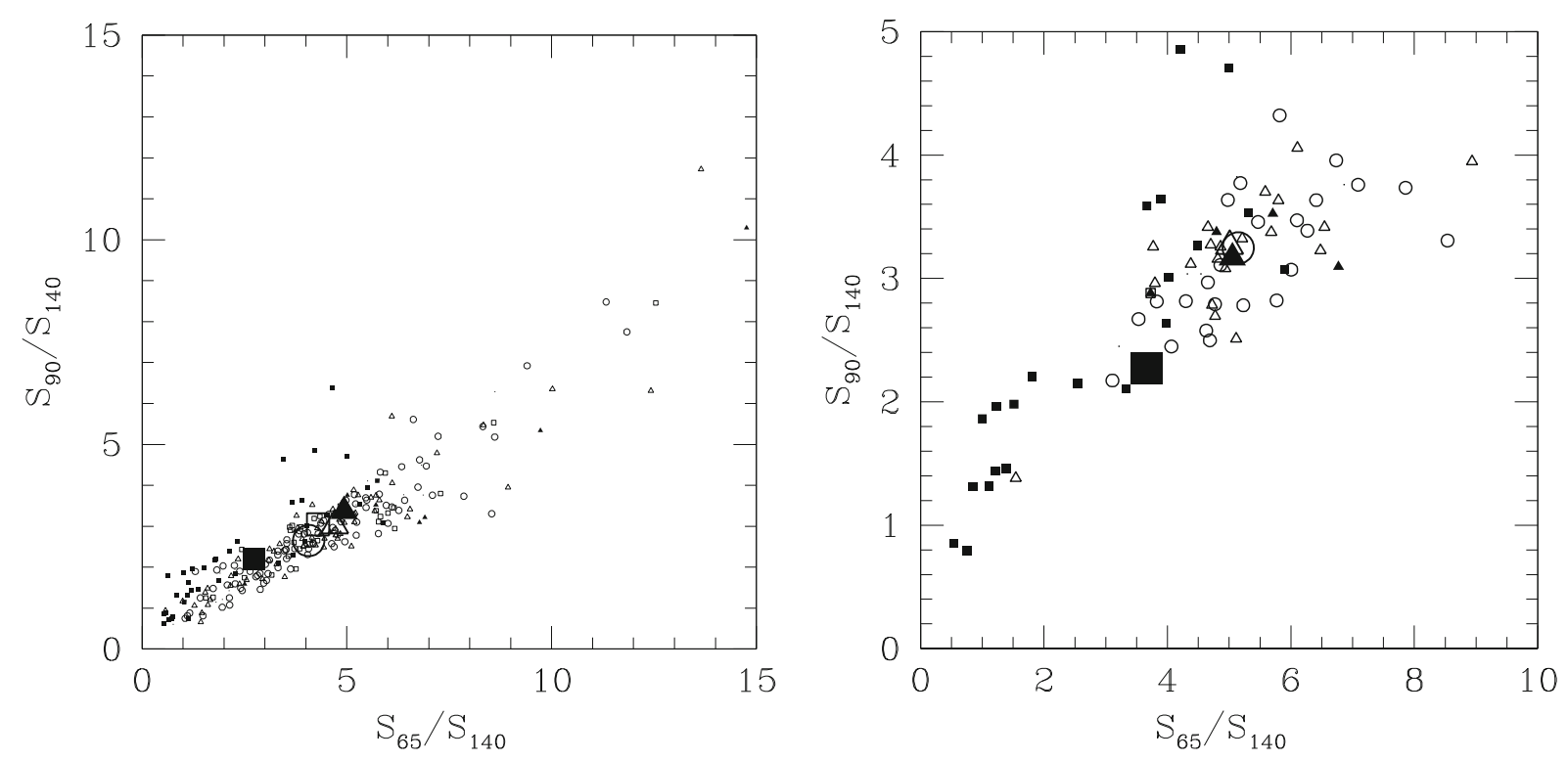

Fig. A.6. Positions of different types of AGB stars in the FIR color-color diagram $S_{65} / S_{140}$ vs. $S_{90} / S_{140}$. All symbols as in Fig. 1 . In the left panel, all sources are presented. In the right panel, only sources with quality flags 3 are included (note difference in scale). 

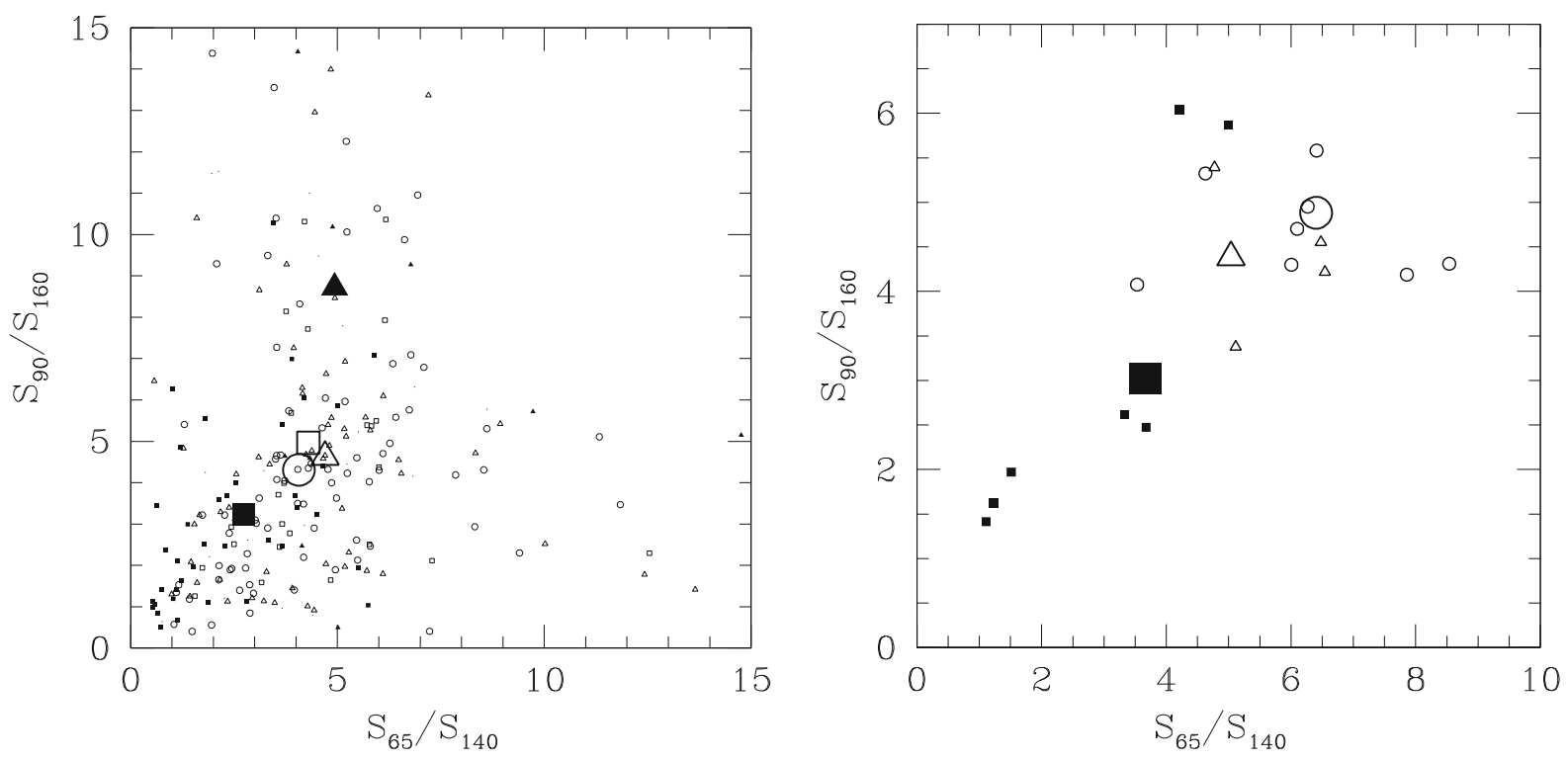

Fig. A.7. Positions of different types of AGB stars in the FIR color-color diagram $S_{65} / S_{140}$ vs. $S_{90} / S_{160}$. All symbols as in Fig. 1 . In the left panel, all sources are presented. In the right panel, only sources with quality flags 3 are included (note difference in scale).
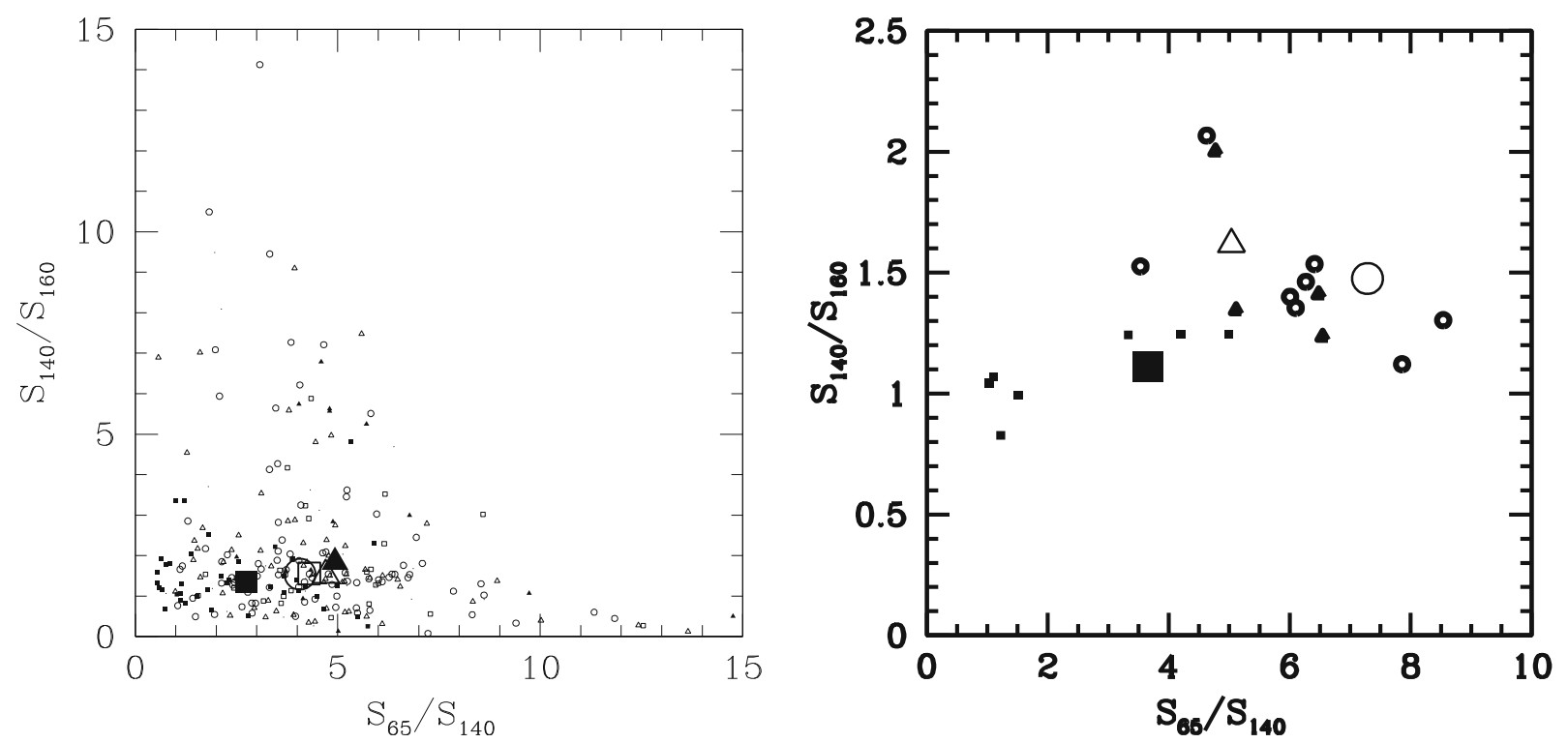

Fig. A.8. Positions of different types of AGB stars in the FIR color-color diagram $S_{65} / S_{140}$ vs. $S_{140} / S_{160}$. All symbols as in Fig. 1 . In the left panel, all sources are presented. In the right panel, only sources with quality flags 3 are included (note difference in scale). 

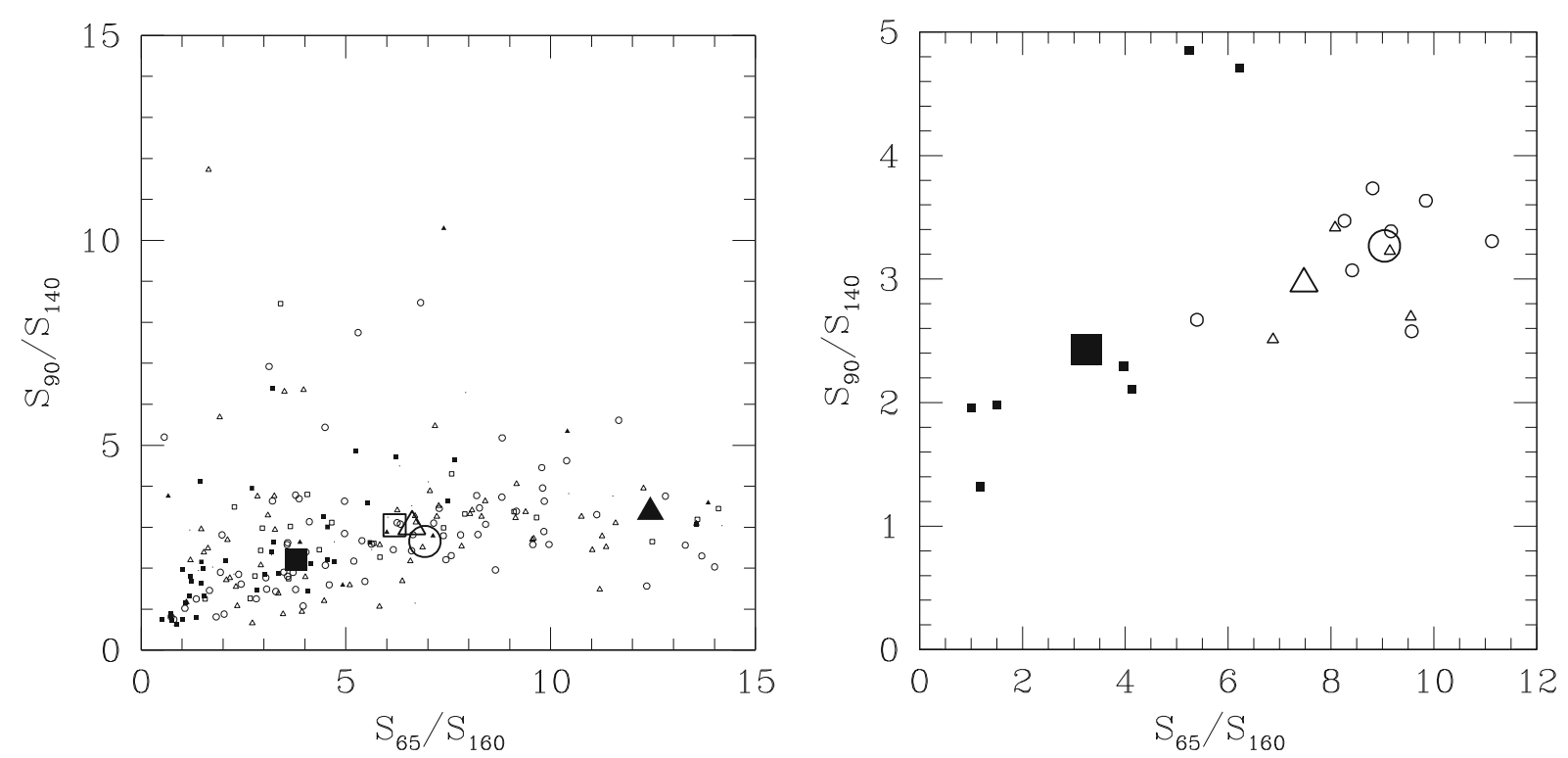

Fig. A.9. Positions of different types of AGB stars in the FIR color-color diagram $S_{65} / S_{160}$ vs. $S_{90} / S_{140}$. All symbols as in Fig. 1. In the left panel, all sources are presented. In the right panel, only sources with quality flags 3 are included (note difference in scale).
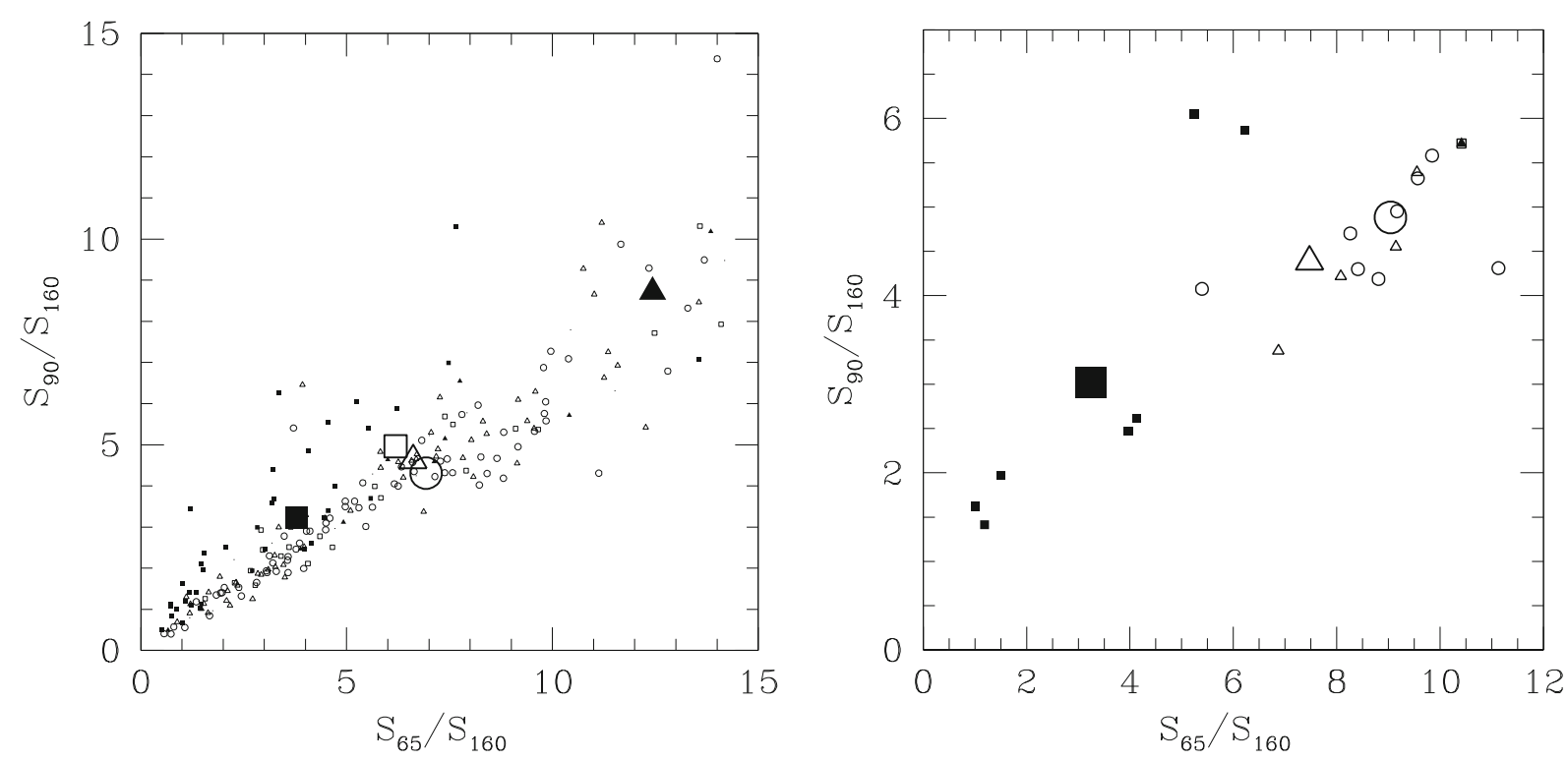

Fig. A.10. Positions of different types of AGB stars in the FIR color-color diagram $S_{65} / S_{160}$ vs. $S_{90} / S_{160}$. All symbols as in Fig. 1 . In the left panel, all sources are presented. In the right panel, only sources with quality flags 3 are included (note difference in scale). 

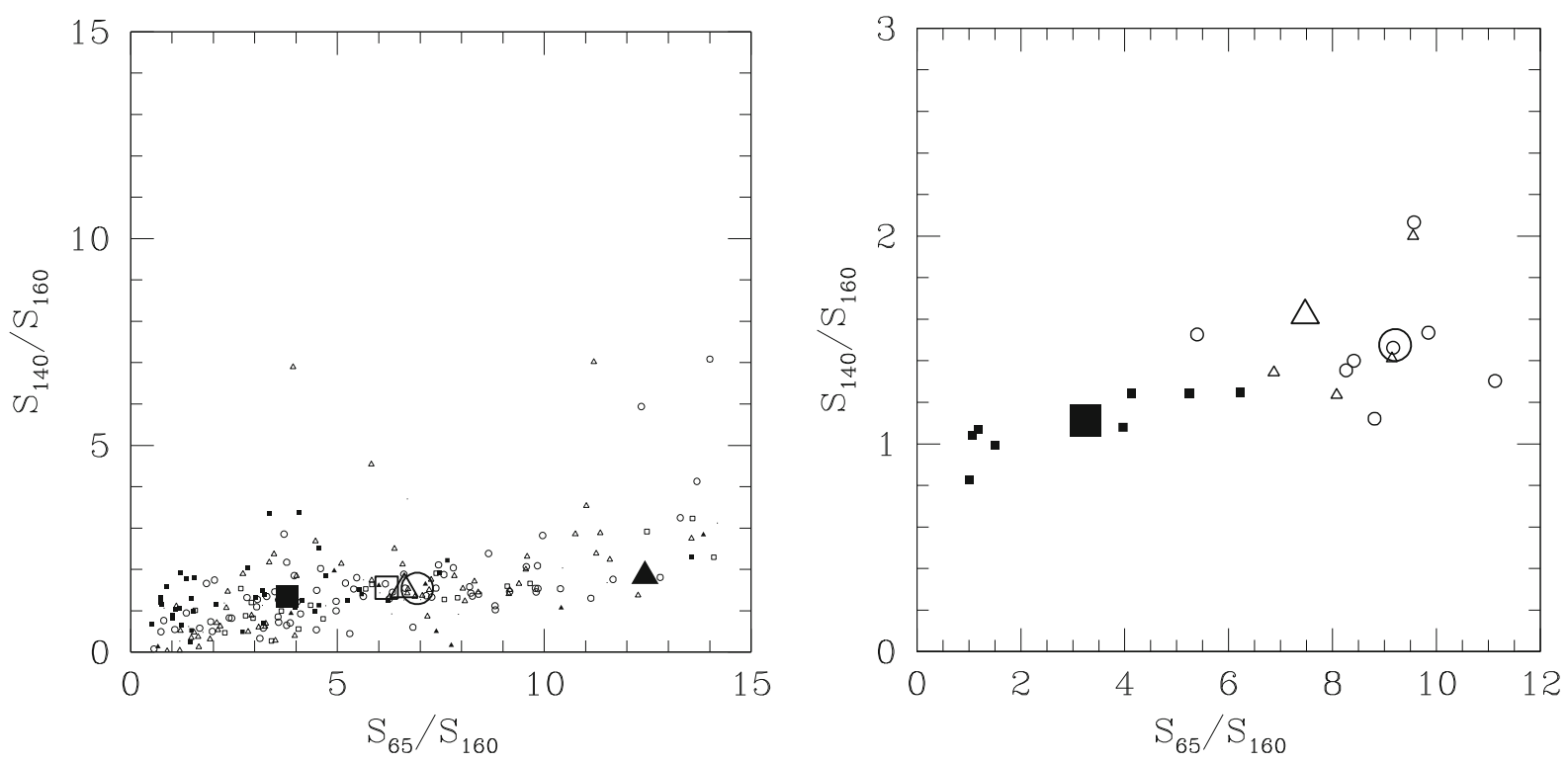

Fig. A.11. Positions of different types of AGB stars in the FIR color-color diagram $S_{65} / S_{160}$ vs. $S_{140} / S_{160}$. All symbols as in Fig. 1. In the left panel, all sources are presented. In the right panel, only sources with quality flags 3 are included (note difference in scale).
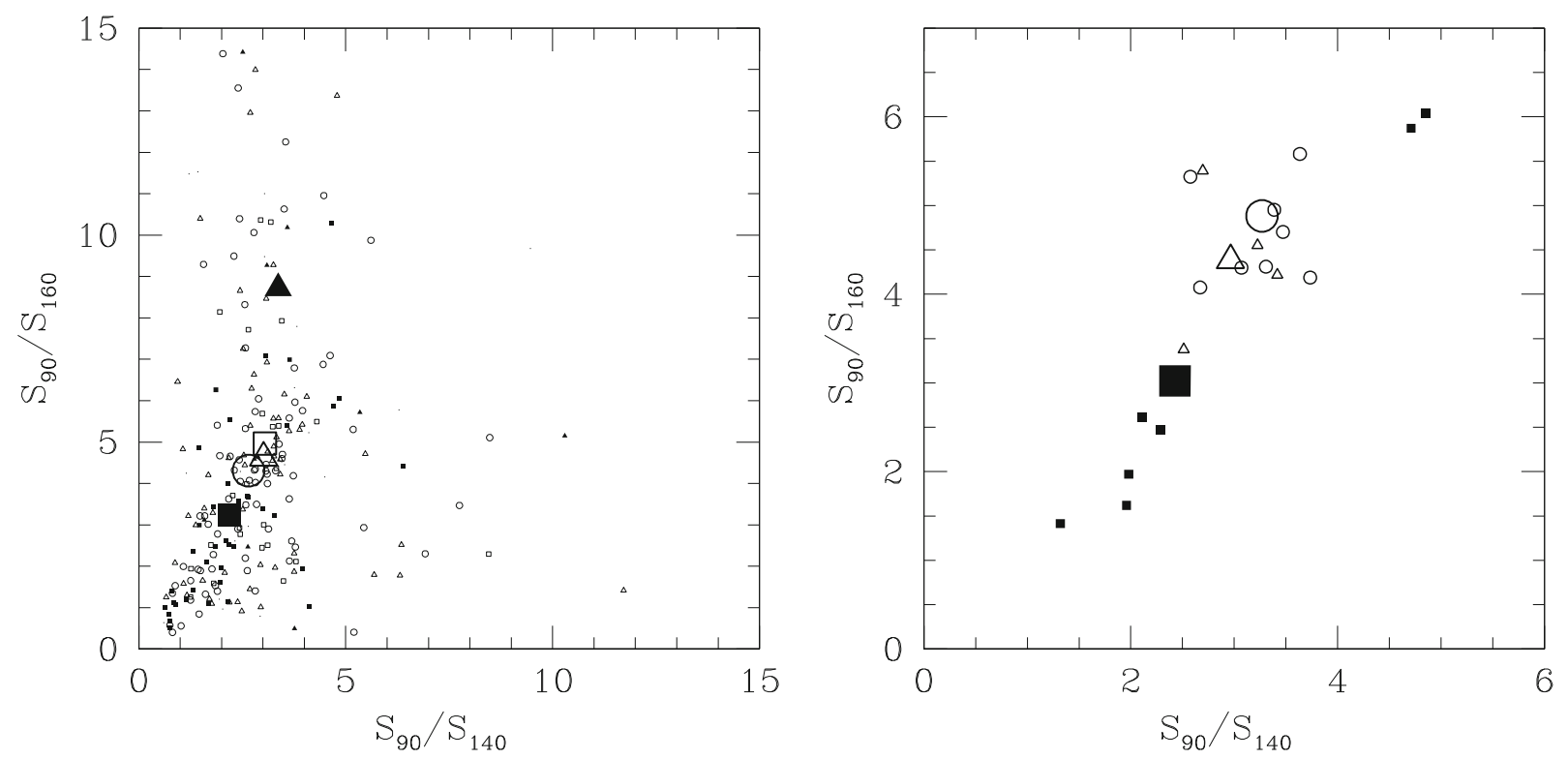

Fig. A.12. Positions of different types of AGB stars in the FIR color-color diagram $S_{90} / S_{140}$ vs. $S_{90} / S_{160}$. All symbols as in Fig. 1 . In the left panel, all sources are presented. In the right panel, only sources with quality flags 3 are included (note difference in scale). 

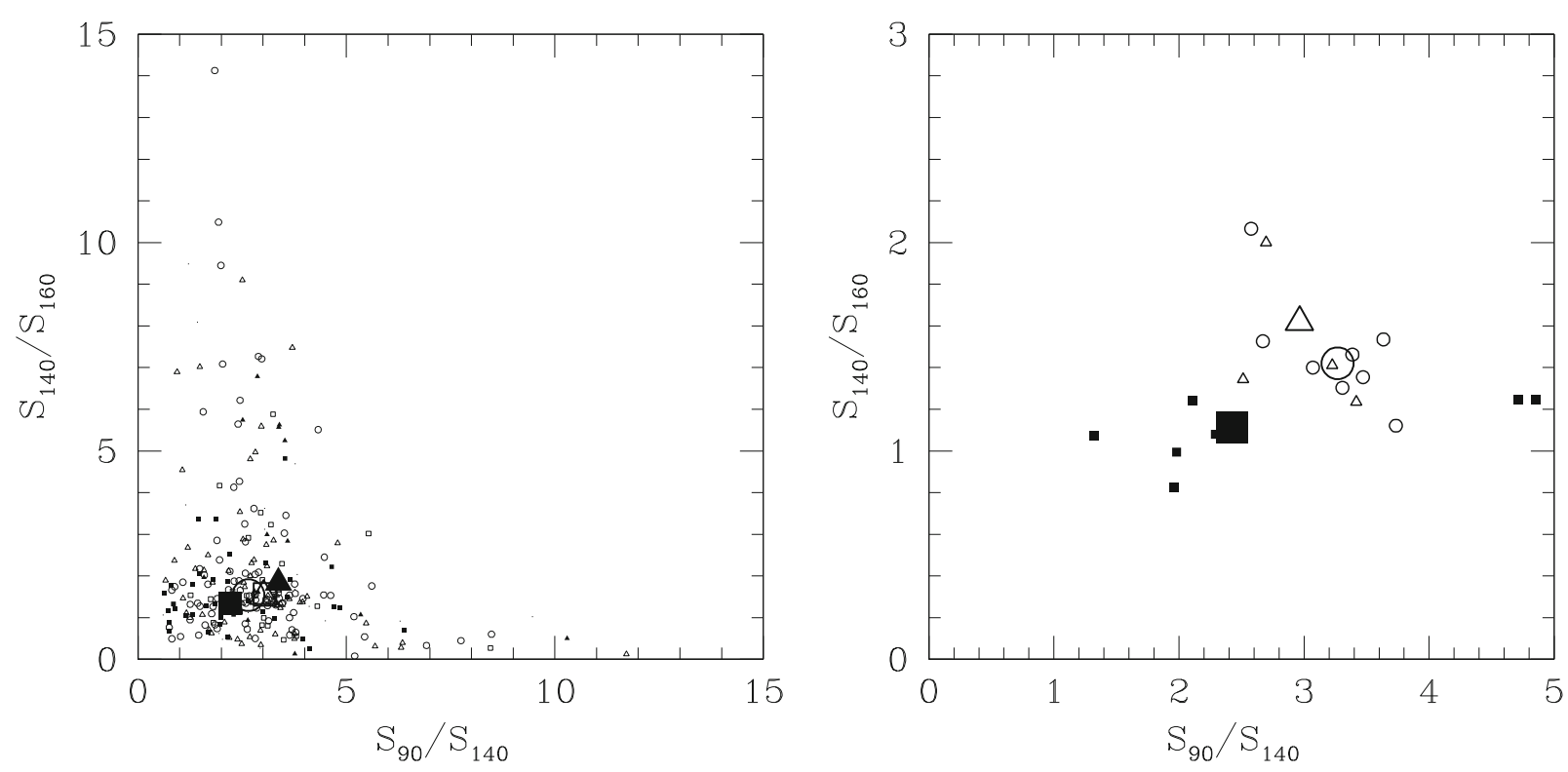

Fig. A.13. Positions of different types of AGB stars in the FIR color-color diagram $S_{90} / S_{140}$ vs. $S_{140} / S_{160}$. All symbols as in Fig. 1. In the left panel, all sources are presented. In the right panel, only sources with quality flags 3 are included (note difference in scale).
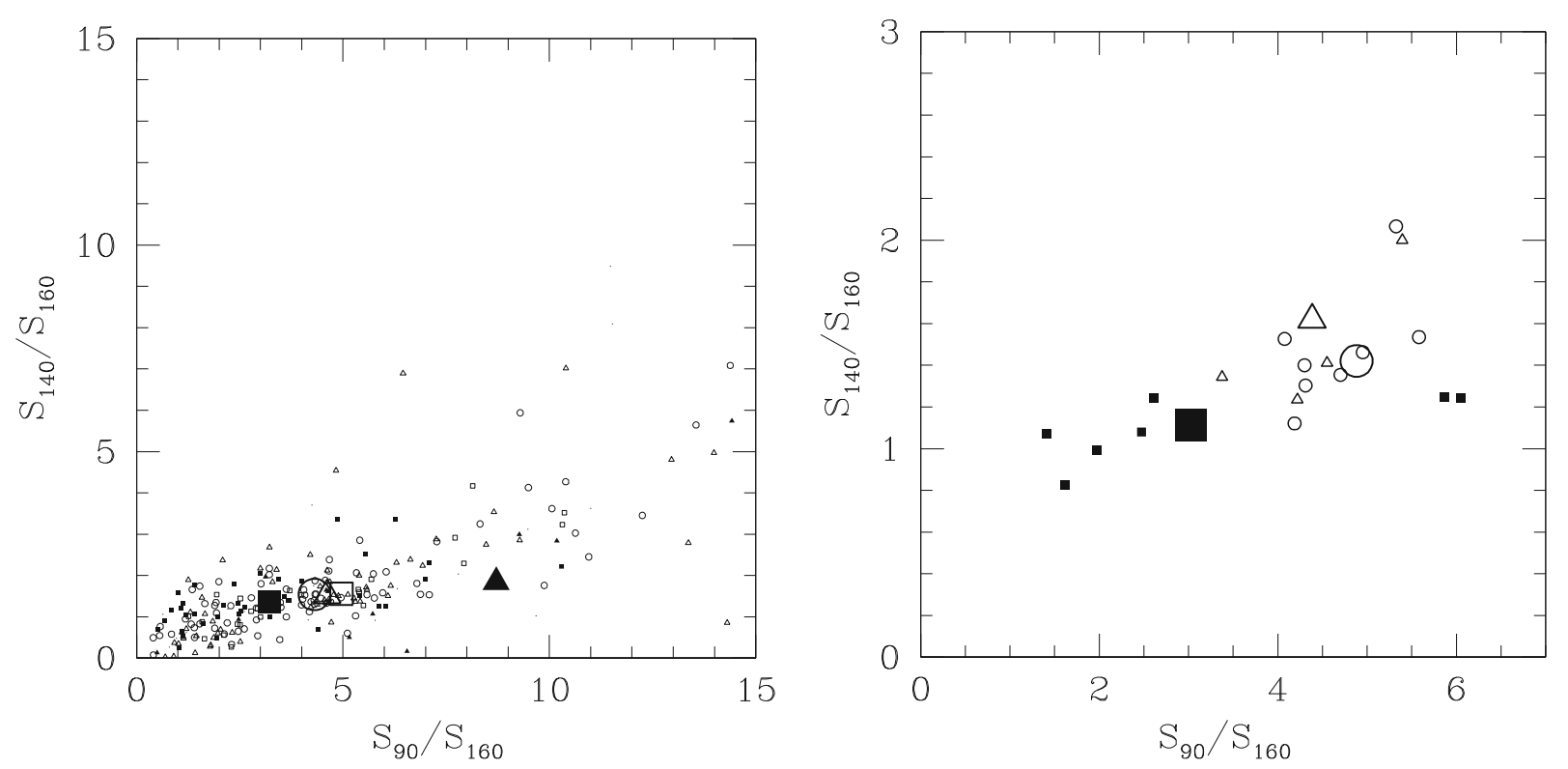

Fig. A.14. Positions of different types of AGB stars in the FIR color-color diagram $S_{90} / S_{160}$ vs. $S_{140} / S_{160}$. All symbols as in Fig. 1 . In the left panel, all sources are presented. In the right panel, only sources with quality flags 3 are included (note difference in scale). 